\title{
Large-Eddy Simulation of Time Evolution and Instability of Highly Underexpanded Sonic Jets
}

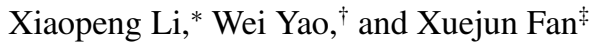 \\ Chinese Academy of Sciences, 100190 Beijing, People's Republic of China
}

\begin{abstract}
DOI: $\underline{10.2514 / 1 . J 054689}$
High-pressure jet injection into quiescent air is a challenging fluid dynamics problem in the field of aerospace engineering. Although plenty of experimental, theoretical, and numerical studies have been conducted to explore this flow, there is a dearth of literature detailing the flow evolution and instability characteristics, which is vital to the mixing enhancement design and jet noise reduction. In this paper, a density-based solver for compressible supersonic flow, astroFoam, is developed based on the OpenFOAM library. Large-eddy simulations of highly underexpanded jets with nozzle pressure ratios from 5.60 to 11.21 at a Reynolds number around $10^{5}$ are carried out with a highresolution grid. A grid-convergence study has been conducted to confirm the fidelity of the large-eddy simulation results. The large-eddy simulation results have also been validated against available literature data in terms of the time-averaged near-field properties of underexpanded jets. The turbulent transition processes are revealed based on the instantaneous flow features and are quantitatively resolved according to the jet penetration and maximum width. The vorticity analysis is conducted to understand the turbulent transition mechanism, and it is found that the vortex stretching term plays a leading role on the distortion of the vortex rings in the near field of the jets. The dominant instability modes of jets, visualized by helicity, are quantitatively revealed based on the spectrum and relative phase of pressure fluctuation. The single helical modes corresponding to a phase angle close to $\pm 180 \mathrm{deg}$ with the $1+1$ helices are dominant for nozzle pressure ratios of 5.60 and 7.47, whereas the complex and multiple helices for the other two higher nozzle pressure ratios are due to the superposition of the single and double helical modes. In addition, the performance of the coarse mesh and different subgrid-scale models on capturing the dominant instability characteristics in large-eddy simulation of underexpanded jets is investigated.
\end{abstract}

\section{Introduction}

$\mathbf{H}$ IGHLY underexpanded jets may be found in a great deal of practical applications, especially in high-speed propulsion fields, such as the air/fuel mixing inside scramjets, the takeoff of rockets, and the landing of space shuttles. Highly underexpanded jets are defined by a nozzle pressure ratio (NPR) beyond 3.85 and are characterized by the presence of a Mach disk in the near-field region. Besides the near-field shock structures, the highly underexpaned jets are also characterized by the unsteady large-scale coherent structures along the jet shear layer. The interactions between the near-field shocks and the coherent structures make the highly underexpanded jet flows complicated. Therefore, the investigation of highly underexpanded jets is an involved subject for both engineering applications and gas dynamics [1-7].

Adamson and Nicholls [1] were the first to present the structure of a highly underexpanded jet into quiescent air. After that, there have been many efforts [2-6] devoted to investigating the properties of highly underexpanded jets. For example, Crist et al. [2] derived the conjunction between Mach disk size and NPR. Donaldson and Snedeker [3] categorized the jets into three major types based on the NPR as subsonic, moderately underexpanded, and highly underexpanded. Chuech et al. [4] investigated the effects of compressibility and turbulence levels at the jet exit on the near-field region of underexpanded turbulent jets experimentally. Wu et al. [5] examined the effects of nozzle exit temperature on the size of the

Received 19 August 2015; revision received 2 March 2016; accepted for publication 10 March 2016; published online 31 May 2016. Copyright $\odot 2016$ by the American Institute of Aeronautics and Astronautics, Inc. All rights reserved. Copies of this paper may be made for personal and internal use, on condition that the copier pay the per-copy fee to the Copyright Clearance Center (CCC). All requests for copying and permission to reprint should be submitted to CCC at www.copyright.com; employ the ISSN 0001-1452 (print) or 1533-385X (online) to initiate your request.

*Post-Doctoral Researcher, State Key Laboratory of High Temperature Gas Dynamics, Institute of Mechanics; 1xpyfy@163.com.

${ }^{\dagger}$ Associate Professor, State Key Laboratory of High Temperature Gas Dynamics, Institute of Mechanics.

${ }^{\ddagger}$ Professor, State Key Laboratory of High Temperature Gas Dynamics, Institute of Mechanics; xfan@imech.ac.cn.
Mach disk and the expansion angle. André et al. [6] studied the expansion, compression, and neutral zones in highly underexpanded jets experimentally. One may also refer to the recent review on underexpanded jets by Franquet et al. [7] for further details. Note that most of these experimental data were obtained by schlieren photography, which is generally insufficient to characterize the unsteady characteristics of underexpanded jets because of low spatial and temporal resolution. There are several computational studies [811] carried out as well to determine the various averaged flow features of highly underexpanded jets at different conditions. For example, Ouellette and Hill [8] studied the jet penetration rates of underexpanded jets using Reynolds-averaged Navier-Stokes (RANS) simulation with a modified $k-\varepsilon$ model. Otobe et al. [9], Menon and Skews [10], and Hatanaka and Saito [11] all investigated the influence of nozzle geometry on the near-field structures of underexpanded sonic jets with RANS simulations. These RANSbased simulations mainly provided the time-averaged flow properties, but the flow evolution and transition mechanism of underexpanded jets are rarely explored. On the other hand, the instantaneous flowfield differs greatly from the ensemble-averaged one due to high levels of turbulent fluctuations in the shear layer. Revealing the unsteady flow characteristics and understanding the transition mechanism of underexpanded jets is therefore of great benefit for practical fuel mixing enhancement design and active flow control.

Powell [12] observed experimentally that supersonic underexpanded jets will produce screech, which is characterized by powerful discrete tones in the frequency spectrum. Over the years, plenty of experimental, theoretical, and numerical studies [13-22] have been carried out to investigate the screech tones of supersonic underexpanded jets, and knowledge on the generation and prediction of the phenomenon has been well gained. In particular, this behavior was generally believed to be controlled by an acoustic feedback loop and related with the instability modes of the jet shear layer. There were several experimental works [23-25] on multimode structures of supersonic underexpanded jets from the viewpoint of shock screech. Gutmark et al. [23] studied the mode switching of supersonic underexpanded jets for NPR $2.0 \sim 15.0$ experimentally. Powell et al. [24] investigated the dominant mode of underexpanded choked circular jets for NPR $2.0 \sim 5.84$ with schlieren photography and 
observed that the mode of jet instability changes successively from an axially symmetric (varicose, toroidal) one to a sinuous (lateral, flapping) one, a helical one, and finally a sinuous one as NPR increases. Panda and Seasholtz [25] measured the shock structure and shock-vortex interaction in underexpanded jets for NPR $1.588 \sim$ 5.75 and found that the evolution of the large-scale turbulent vortices along the shear layer is modulated periodically along the flow direction. However, it is difficult to get an accurate representation of tangential modes of jets using schlieren data, and only limited information about the instantaneous three-dimensional unsteady characteristics of underexpanded jets can be obtained by the twodimensional pictures in the streamwise direction.

Considering the great cost in computing time, direct numerical simulation of supersonic flows in realistic geometries of high Reynolds number is generally unaffordable. On the other hand, largeeddy simulation (LES), which resolves the large scales directly and models the effect of small scales, has the potential to study the flow characteristics of highly underexpanded jets. There has been some LES research work on supersonic conditions [26-28] in the recent years. For example, Kawai and Lele [26], Génin and Menon [27], and Rana et al. [28] all reproduced the flow conditions of a sonic jet in supersonic crossflow (JISC) reported in the experiments by Santiago and Dutton [29] and studied the physics of the jet mixing based on the LES technique. These studies show that the LES technique is capable of capturing salient flow structures and dynamics in supersonic turbulent environments. As for highly underexpanded jets, to the best of our knowledge, papers on the applications of a fully threedimensional LES modeling are very few. Gorle et al. [30] employed a dynamic version of the Vreman model [31] to take into account the effects of the subgrid scales (SGSs) and performed an LES modeling of a highly underexpanded jet at NPR of 30 to verify the jet injection model. Vuorinen et al. [32] provided the first systematic assessment on NPR dependency of highly underexpanded jet structures using a finite-volume-type implicit LES (ILES) technique. The LES results by Vuorinen et al. [32] compared favorably to the experimental data, but they mainly focused on the quasi-steady-state jet structures. The information about the detailed flow evolution and the mechanisms driving the turbulent transition of underexpanded jets is limited. Note that generally no additional SGS modes are implemented in an ILES modeling because the numerical dissipation needed to model the subgrid scales is provided by the numerical methods employed. However, the finite volume methods coupled with the explicit SGS models would be preferred in more complex geometries. As a result, the effects of different SGS models in an explicit LES approach on capturing the key flow characteristics of supersonic jets, such as the helical modes, are worth examining.

In this study, a density-based finite volume supersonic compressible flow solver, astroFoam, is developed in the framework of OpenFOAM computational fluid dynamics (CFD) libraries. A three-dimensional nozzle geometry is employed to capture the selfsustained acoustic loop correctly. The LES modeling of highly underexpanded sonic nitrogen jets at four different NPRs is performed with a high-resolution mesh. The grid independence and convergence are investigated, and the size of the Mach disk is compared with the measurements and literature data to verify the current LES modeling. Then, two aspects about highly underexpanded jets are focused on and studied based on the in-depth analysis of LES data. One is the turbulent transition processes, characteristics, and the possible mechanisms. The other is the dominant instability characteristics of underexpanded jets at quasisteady state. In addition, the effect of grid and the SGS models employed in LES to capture the key flow characteristics of underexpanded jets is investigated.

\section{Numerical Procedure}

The computational study is based on the C++ open-source CFD package OpenFOAM, which contains numerous solvers and utilities covering a wide range of problems. The astroFoam solver employed in the present study is developed based on the standard rhoCentralFoam solver [33] distributed with OpenFOAM v2.3.0.
Vuorinen et al. [32] has developed a similar OpenFOAM solver and applied it to the LES modeling of sonic turbulent jets. Fureby et al. [34], Chapuis et al. [35], and Arisman et al. [36] have also developed the similar compressible OpenFOAM solvers to study the supersonic combustion, gaseous explosions, and the effects of nitric oxide chemistry on hypersonic boundary layers, respectively.

\section{A. Governing Equations and Numerical Methods}

The filtered Favre-averaged conservation equations of mass, momentum, energy, and species concentration used in astroFoam are defined as

$$
\begin{gathered}
\frac{\partial \bar{\rho}}{\partial t}+\frac{\partial \bar{\rho} \tilde{u}_{i}}{\partial x_{i}}=0 \\
\frac{\partial \bar{\rho} \tilde{u}_{i}}{\partial t}+\frac{\partial \bar{\rho} \tilde{u}_{i} \tilde{u}_{j}}{\partial x_{j}}=-\frac{\partial \bar{p}}{\partial x_{i}}+\frac{\partial \tilde{\tau}_{i j}}{\partial x_{j}}-\frac{\partial \tau_{i j}^{\mathrm{sgs}}}{\partial x_{j}}+\frac{\partial D_{i j}^{\mathrm{sgs}}}{\partial x_{j}} \\
\frac{\partial \tilde{\rho} \tilde{h}_{s}}{\partial t}+\frac{\partial \tilde{\rho} \tilde{u}_{j} \tilde{h}_{s}}{\partial x_{j}}=\frac{\partial \bar{p}}{\partial t}+\frac{\partial \tilde{u}_{j} \tilde{\tau}_{i j}}{\partial x_{i}}-\frac{\partial \bar{q}_{i}}{\partial x_{i}}-\frac{\partial H_{i}^{\mathrm{sgs}}}{\partial x_{i}}+\frac{\partial \sigma_{i}^{\mathrm{sgs}}}{\partial x_{i}} \\
\frac{\partial \bar{\rho} \tilde{Y}_{k}}{\partial t}+\frac{\partial \bar{\rho} \tilde{u}_{j} \tilde{Y}_{k}}{\partial x_{j}}=\frac{\partial}{\partial x_{j}}\left(\bar{\rho} D_{\mathrm{km}} \frac{\partial \tilde{Y}_{k}}{\partial x_{j}}\right)-\frac{\partial \Phi_{k, j}^{\mathrm{sgs}}}{\partial x_{j}}-\frac{\partial \theta_{k, j}^{\mathrm{sgs}}}{\partial x_{j}} \\
\bar{p}=\bar{\rho} R \tilde{T}+R_{u} \sum_{k=1}^{N} T^{\mathrm{sgs}}
\end{gathered}
$$

where the overbar ("-") and tilde (" $\sim$ ") mean the cell and Favre average, respectively; $\rho$ is the density; $u_{i}$ is the velocity in the $x_{i}$ direction; $p$ is the pressure; $\tau_{i j}$ is the viscous stress tensor; $h_{s}$ is the sensible enthalpy per unit mass; $q_{i}$ is the heat flux vector; $Y_{k}$ is the species mass fraction; $D_{\mathrm{km}}$ is the equivalent binary mass diffusivity; $T$ is the temperature; $R$ is the gas constant of gas mixture; and $R_{u}$ is the universal gas constant.

The preceding equations are solved using the compressible solver astroFoam, which is modified from the basic rhoCentralFoam solver. The rhoCentralFoam solver was first developed by Greenshields et al. [33] and has been successfully employed in modeling the supersonic flows by solving the convection-diffusion equation using a secondorder semidiscrete, nonstaggered Kurganov and Tadmor (KT) central-upwind scheme [37]. However, the rhoCentralFoam solver is limited to single-species nonreacting flows in its standard form. Multiple species transport and multicomponent diffusion [Eq. (4)] are added in the astroFoam solver to model more realistic mixing and reacting flows. The energy equation [Eq. (3)] is solved for sensible enthalpy in astroFoam instead of total energy in rhoCentralFoam to include the chemical reaction and species transport terms more easily. In astroFoam, the convection-diffusion equation is also solved by the semidiscrete KT scheme for shock-capturing and turbulence resolving. A normalized variable diagram scheme $[38,39]$ is applied to reconstruct the primitive values at faces to obtain the higher-order accuracy. The so-called "minmod" limiter is used during the reconstruction to ensure the numerical stability. Time integration is carried out using the Crank-Nicholson scheme [39], which is secondorder in time.

The thermodynamic and transport properties of individual species, such as the enthalpy per unit mass $\left(h_{k}\right)$ and the specific heat at constant pressure $\left(c_{p k}\right)$, are calculated based on NIST-JANAF thermophysical and transport database [40]. The dynamic viscosity $\mu_{k}$ is computed by Sutherland's law. The Schmidt number $S c$ and the 
turbulent Prandtl number $\operatorname{Pr}_{t}$ in the species concentration and energy equations are assumed to be constant of 1.0 .

\section{B. Explicit Subgrid-Scale Model}

Note that all the subgrid-scale terms in Eqs. (1-5), denoted with a "sgs" superscript, are unclosed and require specific modeling. In particular, the SGS viscous stress term $D_{i j}^{\mathrm{sgs}}$, SGS viscous diffusion term $\sigma_{i}^{\text {sgs }}$, SGS species diffusive fluxes term $\theta_{k, j}^{\text {sgs }}$, and SGS speciestemperature correlation term $T^{\mathrm{sgs}}$ correspond to $D_{i j}^{\mathrm{sgs}}=\bar{\tau}_{i j}-\tilde{\tau}_{i j}$, $\sigma_{i}^{\mathrm{sgs}}=\widetilde{u_{j} \tau_{i j}}-\tilde{u}_{j} \tilde{\tau}_{i j}, \quad \theta_{k, j}^{\mathrm{sgs}}=\bar{\rho} \widetilde{V_{k, j} Y_{k}}-\bar{\rho} \tilde{V}_{k, j} \tilde{Y}_{k}, \quad$ and $\quad T^{\mathrm{sgs}}=$ $\bar{\rho}\left(\widetilde{Y_{k} T}-\tilde{Y}_{k} \tilde{T}\right)$, respectively, and are found to be small and generally neglected in the previous studies [27,41-43]. Another unclosed term in the energy equation is SGS energy fluxes term $H_{i}^{\text {sgs }}=$ $\left(\bar{\rho} \widetilde{E_{t} u_{i}}-\bar{\rho} \tilde{E}_{t} \tilde{u}_{i}\right)+\left(\overline{p u_{i}}-\bar{p} \tilde{u}_{i}\right)$, which is modeled based on a linear eddy diffusivity assumption as

$$
H_{j}^{\mathrm{sgs}}=-\bar{\rho} \frac{\nu_{t}}{P r_{t}} \frac{\partial \tilde{H}}{\partial x_{j}}=-\bar{\rho} \frac{\nu_{t}}{P r_{t}}\left(\frac{\partial \tilde{h}}{\partial x_{j}}+\tilde{u}_{i} \frac{\partial \tilde{u}_{i}}{\partial x_{j}}+\frac{\partial k^{\mathrm{sgs}}}{\partial x_{j}}\right)
$$

where $\nu_{t}$ is the eddy viscosity. The SGS species fluxes term $\Phi_{k, j}^{\mathrm{sgs}}=$ $\bar{\rho} \widetilde{u_{j} Y_{k}}-\bar{\rho} \tilde{u}_{j} \tilde{Y}_{k}$ is also modeled using the eddy diffusivity assumption as

$$
\Phi_{k, i}^{\mathrm{sgs}}=-\bar{\rho} \frac{\nu_{t}}{S c_{t}} \frac{\partial \tilde{Y}_{k}}{\partial x_{i}}
$$

Another unclosed term in the momentum equation is the SGS stress term, $\tau_{i j}^{\mathrm{sgs}}=\bar{\rho}\left(\widetilde{u_{i} u_{j}}-\tilde{u}_{i} \tilde{u}_{j}\right)$. The dissipative scales are generally not resolved in LES, and so the main role of the subgrid stress model is to extract energy from the resolved scales, thus modeling the drain associated with the energy cascade. This can be accomplished with an eddy-viscosity model, where $\tau_{i j}^{\text {sgs }}$ is given in terms of the SGS turbulent kinetic energy as

$$
\tau_{i i j}^{\mathrm{sgs}}-\frac{2 \delta_{i j}}{3} \bar{\rho} k^{\mathrm{sgs}}=-2 v_{t} \bar{\rho}\left[\tilde{S}_{i j}-\frac{\delta_{i j}}{3} \tilde{S}_{k k}\right]
$$

where $\tilde{S}_{i j}$ is the strain rate tensor for the resolved scale and is defined by

$$
\tilde{S}_{i j}=\frac{1}{2}\left(\frac{\partial \tilde{u}_{i}}{\partial x_{j}}+\frac{\partial \tilde{u}_{j}}{\partial x_{i}}\right)
$$

To derive the eddy viscosity $\nu_{t}$, two different subgrid-scale models are employed in the present study. The first one is the Smagorinsky model, which is proposed by Smagorinsky [44] first and is further developed by Yoshizawa [45] and Erlebacher et al. [46]. It can be expressed as follows:

$$
\begin{gathered}
v_{t}=C_{R} \bar{\Delta}^{2}|\tilde{S}| \\
\tau_{k k}^{\mathrm{sgs}}=2 \bar{\rho} k^{\mathrm{sgs}}=2 C_{I} \bar{\Delta}^{2} \bar{\rho}|\tilde{S}|^{2}
\end{gathered}
$$

where $|\tilde{S}|=\sqrt{2 \tilde{S}_{i j} \tilde{S}_{i j}}, \bar{\Delta}$ is the filter width, and $C_{R}$ and $C_{I}$ are Smagorinsky coefficients. Note that the Smagorinsky model is derived from the simplifying assumption that the subgrid scales are in equilibrium and dissipate entirely and instantaneously all the energy received from the resolved scales. However, nonequilibrium conditions commonly occur, such as in free shear-layer flows considered in this work, separating and reattaching flows, and wall-bounded flows like pipe and channel flows. Therefore, a one-equation model [47-51], in which a transport equation for the subgrid turbulent kinetic energy $k^{\mathrm{sgs}}$ is provided to account for the nonequilibrium effects, is applied in the current work, and the corresponding expression is

$$
\begin{gathered}
\frac{\partial \bar{\rho} k^{\mathrm{sgs}}}{\partial t}+\frac{\partial \bar{\rho} \tilde{u}_{j} k^{\mathrm{sgs}}}{\partial x_{j}}=\frac{\partial}{\partial x_{j}}\left[\bar{\rho}\left(\frac{\nu_{t}}{P r_{t}}+\nu\right) \frac{\partial k^{\mathrm{sgs}}}{\partial x_{j}}\right] \\
-\tau_{i j}^{\mathrm{sgs}} \frac{\partial \tilde{u}_{i}}{\partial x_{j}}-C_{\varepsilon} \frac{\bar{\rho}\left(k^{\mathrm{sgs}}\right)^{3 / 2}}{\bar{\Delta}} \\
v_{t}=C_{\nu} \bar{\Delta} \sqrt{k^{\mathrm{sgs}}}
\end{gathered}
$$

where $C_{\nu}$ and $C_{\varepsilon}$ are model constants. A study of the performance of different SGS models in channel flows by Fureby et al. [50] shows that the one-equation model has advantages in modeling the transitional flows or flows with large-scale unsteadiness compared to the "zero" equation models (e.g., Smagorinsky). Therefore, the one-equation model is used throughout the current work, whereas the effect of the Smagorinsky model is only examined in Sec. IV.F.

\section{Computational Domain and Flow Conditions}

Figure 1 shows the schematic of the computational domain, which mainly consists of a box of size $50 \times 100 \times 50 \mathrm{~mm}$ in the $x, y$, and $z$ directions, respectively. Previous studies $[\underline{12}-18,24]$ indicated that the sound waves originated in the downstream will propagate upstream to change the initial shear-layer structures at the nozzle exit, which will influence the development of the jet shear layer in the downstream further. To capture this acoustic feedback phenomenon correctly, a convergent nozzle geometry is included in the upstream part of the computational domain. The nitrogen jet (mas fraction $Y_{\mathrm{N} 2}=1.0$ ) in the high-pressure nozzle (with total pressure $P_{0}$ and total temperature $T_{0}$ ) is injected into the quiescent air (with stastic pressure $P_{\infty}$, and stastic temperature $T_{\infty}$ ) from a contoured round orifice of $2.0 \mathrm{~mm}$ in diameter $(D=2.0 \mathrm{~mm})$. The height of the nozzle is $20 \mathrm{~mm}$. The three-dimensional schematic of the nozzle is presented in Fig. 1c.

In the present work, the quiescent air is the mixture of nitrogen 0.76699 and oxygen 0.23301 by weight, and initially the temperature, pressure, density, and velocity are respectively uniform (i.e., $\quad T_{\infty}=300 \mathrm{~K}, \quad P_{\infty}=101,325 \mathrm{~Pa}, \quad \rho_{\infty}=1.17 \mathrm{~kg} / \mathrm{m}^{3}$, $U_{\infty}=0$ ). Four different simulations are carried out for NPR $=5.60,7.47,9.34$, and 11.21, which are all typically highly underexpanded jets according to the definition by Donaldson and Snedeker [3]. The flow velocities are close to the sonic speed at the nozzle exit. The Reynolds number at the nozzle exit is about $R e \sim 10^{5}$. The details of the simulation conditions are presented in

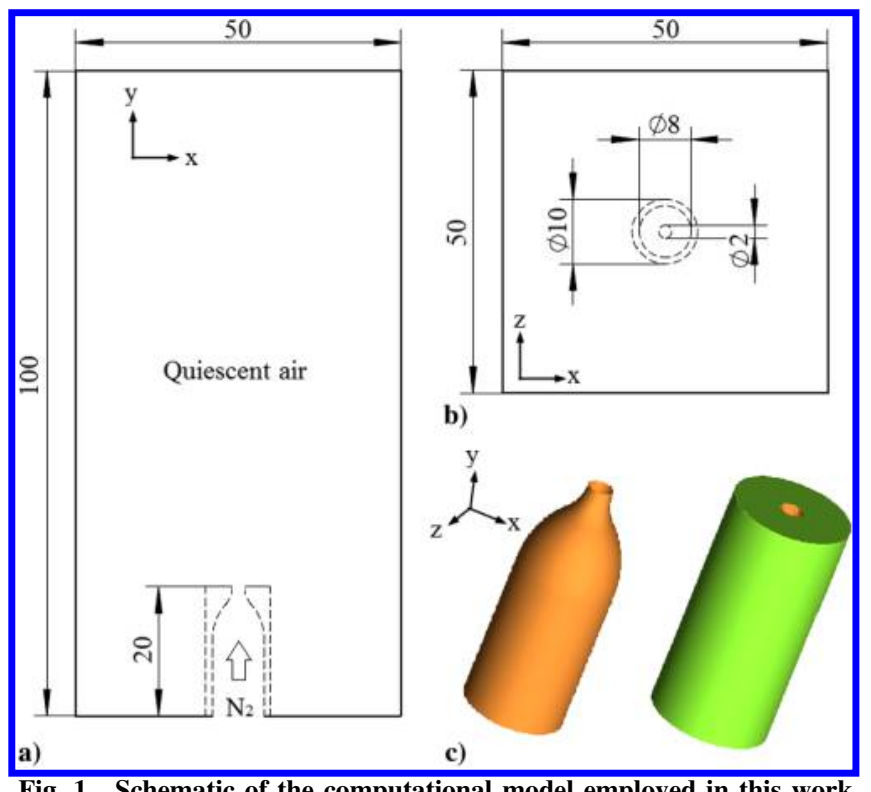

Fig. 1 Schematic of the computational model employed in this work (units in millimeters): a) cross-streamwise view, b) top view, and c) threedimensional schematic of the nozzle. 
Table 1. The inflow at the nozzle inlet is subsonic; thus, the stagnation condition is employed for temperature and pressure, whereas a zerogradient condition is used for velocity. All walls are treated as no-slip, adiabatic conditions. At the top of the computational domain and at the four free surfaces, an open boundary condition is applied (i.e., all flow parameters are treated as zero-gradient for outflow and fixed ambient values in case of backflow).

The integral time scale can be defined using $D$ and the maximum velocities in the near field as $t_{0}=D / 2 U_{1} \approx 2.5 \times 10^{-6} \mathrm{~s}$. In the present study, the pressure and temperature fields are initialized using a linear function, shown in Fig. 2, which is similar to that used by La Vuorinen et al. [32] in the LES modeling of similar high-pressure jet flows. The flow inside the nozzle reached a quasi-steady state around $t \approx 80 t_{0}$, when the nozzle exit pressure $P_{1}$ is $0.3 \mathrm{MPa}$ for NPR $=5.60$. The flow-through time (FTT) for the jets washing out the computational domain is about $0.5 \mathrm{~ms}=200 t_{0}$; thus, the total simulation duration is set as 4FTT $=2.0 \mathrm{~ms}=800 t_{0}$, which is four times the value used in [32] to ensure a long-enough quasi-steady period for turbulent statistics. The instantaneous results are saved every $2 t_{0}$, and turbulent statistics are collected for the last three flowthrough times ( $200 t_{0} \sim 800 t_{0}$, total of 300 time steps).

\section{Computational Mesh}

Previous studies [26-28,30,32] indicated that the spatial resolutions in LES of supersonic jets need to be rather high. The hexahedral, block-structured grid employed in the present study is presented in Fig. 3. The grid contains a refinement region of high resolution, covering the jet core and the jet shear layers. In the refinement region, two levels of grid resolution (i.e., fine and coarse) are used to characterize the grid dependence of the solution. The fine and coarse meshes contain about 27.3 and 13.0 million cells, respectively. The meshing philosophy is as follows.

The radial cell size on the edge of the jet orifice for the [fine] (coarse) mesh is $[0.01 \mathrm{~mm}](0.01 \mathrm{~mm})$, and the resolution decreases to a maximum cell size of $[0.1 \mathrm{~mm}](0.23 \mathrm{~mm})$ at a distance of $5.0 \mathrm{~mm}$ and to $[0.2 \mathrm{~mm}](0.3 \mathrm{~mm})$ at a distance of $10.0 \mathrm{~mm}$ from the nozzle centerline. This region covers the main area of interest in this study including the jet core and the developing shear layer. The cell size is then increased to a maximum of $[1.0 \mathrm{~mm}](1.0 \mathrm{~mm})$ at the edge of the computational domain, as shown in Fig. 3b. The mesh in the streamwise direction is extruded upward with an initial cell size of [0.03 $\mathrm{mm}](0.04 \mathrm{~mm})$ and a maximum of $[0.08 \mathrm{~mm}](0.1 \mathrm{~mm})$ up to $10.0 \mathrm{~mm}$ height from the nozzle exit. Above this height, the mesh is stretched to a maximum cell size of $[0.3 \mathrm{~mm}](0.4 \mathrm{~mm})$ up to $70.0 \mathrm{~mm}$ from the nozzle exit, and then the cell size is increased to a maximum of $[0.5 \mathrm{~mm}](0.5 \mathrm{~mm})$ at the exit of the computational domain to introduce additional dissipation and to avoid wave reflections from the boundary, as shown in Fig. 3a. The grid for the nozzle is also carefully arranged. The cell size in the central part of the nozzle is $[0.017 \mathrm{~mm}](0.024 \mathrm{~mm})$ in the transverse direction and then decreases to $[0.01 \mathrm{~mm}](0.01 \mathrm{~mm})$ at the edge of the jet orifice shown in Fig. 3c. The cell size in the nozzle in the streamwise direction is $[0.2 \mathrm{~mm}](0.3 \mathrm{~mm})$, and the resolution starts to increases at a distance of $3.0 \mathrm{~mm}$ from the nozzle exit, reaching a minimum cell size of [0.03 $\mathrm{mm}](0.04 \mathrm{~mm})$ at the nozzle exit, as shown in Fig. 3a. As a summary, Table 2 lists the grid resolutions in the near field for the

Table 1 Flow parameters and simulation conditions

\begin{tabular}{lcccc}
\hline \hline Property & Case 1 & Case 2 & Case 3 & Case 4 \\
\hline Mach number at nozzle exit $\left(M_{1}\right)$ & 1.0 & 1.0 & 1.0 & 1.0 \\
Static pressure at nozzle exit $\left(P_{1}\right), \mathrm{MPa}$ & 0.3 & 0.4 & 0.5 & 0.6 \\
Stagnation pressure $\left(P_{0}\right), \mathrm{MPa}$ & 0.57 & 0.76 & 0.95 & 1.14 \\
Static temperature at nozzle exit $\left(T_{1}\right), \mathrm{K}$ & 300.0 & 300.0 & 300.0 & 300.0 \\
Density at nozzle exit $\left(\rho_{1}\right), \mathrm{kg} / \mathrm{m}^{3}$ & 3.37 & 4.49 & 5.61 & 6.74 \\
Velocity at nozzle exit $\left(U_{1}\right), \mathrm{m} / \mathrm{s}$ & 353.1 & 353.1 & 353.1 & 353.1 \\
Reynolds number $R e_{1}$ at nozzle exit $\left(\times 10^{5}\right)$ & 1.36 & 1.82 & 2.27 & 2.73 \\
Static pressure ratio $\left(P_{1} / P_{\infty}\right)$ & 2.96 & 3.95 & 4.93 & 5.92 \\
NPR $\left(P_{0} / P_{\infty}\right)$ & 5.60 & 7.47 & 9.34 & 11.21 \\
NDR $\left(\rho_{1} / \rho_{\infty}\right)$ & 2.87 & 3.83 & 4.79 & 5.75 \\
\hline \hline
\end{tabular}

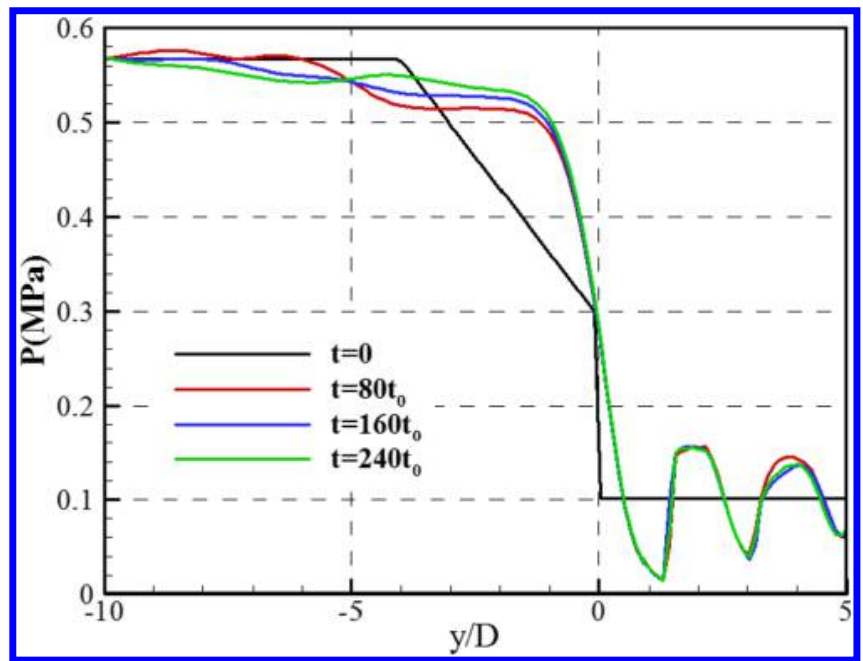

Fig. 2 Time development of pressure profile along the centerline for $\mathrm{NPR}=5.60$. The quasi-steady state is reached at about $t \approx 80 t_{0}$.

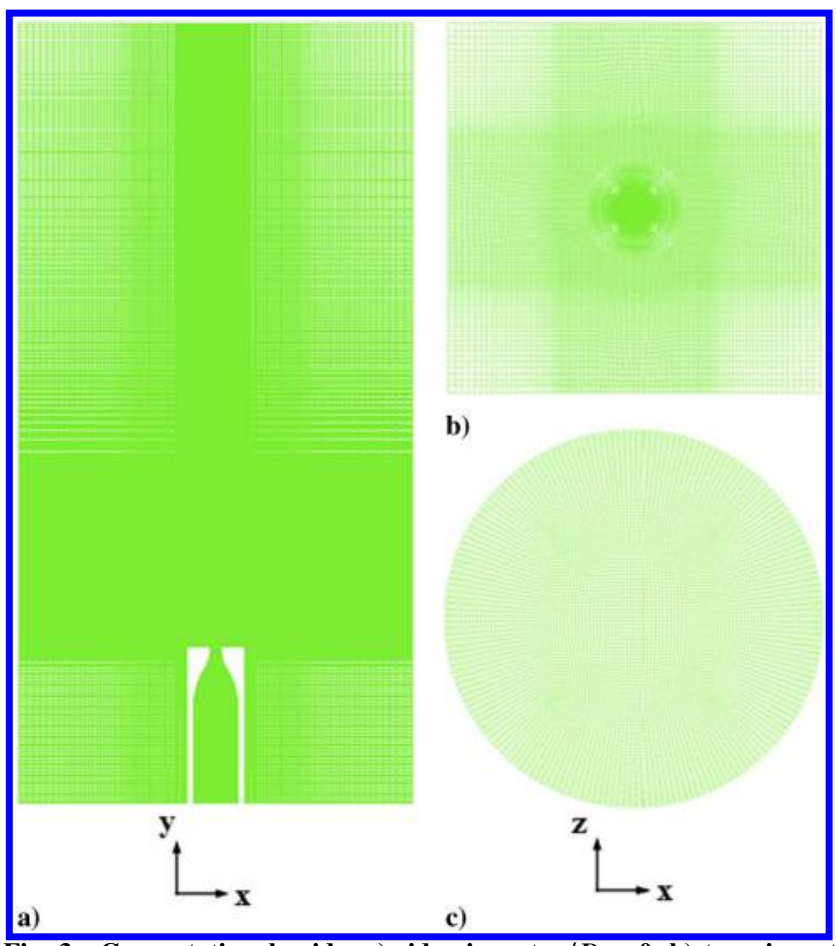

Fig. 3 Computational grids: a) side view at $z / D=0$, b) top view at $y / D=0$, and c) close-up view at nozzle exit.

coarse and fine meshes. As can be seen, the grid resolution employed here is similar to that used in the previous LES of supersonic jets $[28,30,32,52]$.

The computational time step is approximately $\Delta t \approx 1.37 \times 10^{-8} \mathrm{~s}$ $\left(\Delta t \cdot a / D=2.42 \times 10^{-3}\right.$, where $a$ is the speed of sound at the nozzle exit), which gives a maximum Courant-Friedrichs-Lewy number of

Table 2 Grid resolution comparison in the near field $(r / D$ : $-1.5 \sim 1.5 ; y / D: 0 \sim 5 D)$ of supersonic jets

\begin{tabular}{lccccccc}
\hline \hline Grid & $D, \mathrm{~mm}$ & $\Delta r_{\min } \Delta r_{\max }$ & $\Delta y_{\min } \Delta y_{\max }$ & $R e$ & Total $\left(\times 10^{6}\right)$ \\
\hline Fine & 2.0 & $D / 200$ & $D / 52$ & $D / 67$ & $D / 25$ & $\sim 10^{5}$ & 27.3 \\
Coarse & 2.0 & $D / 200$ & $D / 25$ & $D / 50$ & $D / 20$ & $\sim 10^{5}$ & 13.0 \\
Gorle et al. [30] & 2.0 & $D / 100$ & $D / 50$ & $D / 100$ & $D / 25$ & $\sim 10^{5}$ & 17.4 \\
La Vuorinen & 1.4 & $D / 70$ & $D / 50$ & $D / 35$ & $D / 25$ & $\sim 10^{5}$ & 12.0 \\
et al. [32] & & & & & & & \\
Dauptain et al. [52] & 25.4 & $D / 35$ & $D / 30$ & $D / 35$ & $D / 30$ & $\sim 10^{6}$ & 22.0 \\
Rana et al. [28] & 4.0 & $D / 33$ & $D / 33$ & $D / 33$ & $D / 33 \sim 10^{4}$ & 9.2 \\
\hline \hline
\end{tabular}


0.6. This time step is in the same order as the value that Kawai and Lele [26] and Génin and Menon [27] used in their LES of a sonic JISC. The parallel computation is accomplished in the parallel framework of the message passing interface OpenMPI on an Inspur TS10000 high-performance cluster. All together, 96 cores were used for the present simulations, and about two calendar weeks were cost for each case.

\section{Numerical Validation}

\section{A. Mesh Independence and Convergence}

A grid-convergence study is conducted to establish the fidelity of the LES results. The simulation of the highly underexpanded jet at $\mathrm{NPR}=5.60$ has been carried out by using the two different grids (i. e., the coarse and fine meshes). First, it is important to capture the quasi-periodic shock structure. Figure 4 a shows the mean density profile along the jet centerline. For both the coarse and fine meshes, the shock structures in the near fields of the jets $(y / D<10)$ are well resolved. The turbulence properties of the jets also need taking great concern. The radial profiles of the mean streamwise velocity are compared for the two mesh resolutions in Fig. 4b. The overall trends are similar for the two profiles, but the coarse mesh seems to, as expected, restrain the development of turbulence because of larger grid dissipation, which is indicated by a relatively narrower jet boundary.

Figure 5a shows the time history of pressure at NPR of 5.60 for the fine mesh. After one FTT, the pressure fluctuates around its mean value, implying that the flow is fully developed. Sampling of data for evaluation of first-order statistics and spectrum analysis is initiated at $t=200 t_{0}$. The predicted turbulence properties obtained for the two meshes are compared based on the spectrum of pressure fluctuation in Fig. 5b. The two signals are reasonably matched, especially the two discrete dominant peaks. The first dominant peak of frequency is $37.086 \mathrm{kHz}$ for the fine mesh and switches to an approximate value of $35.761 \mathrm{kHz}$ for the coarse mesh. Meanwhile, the higher peak of frequency is $45.695 \mathrm{kHz}$ for the fine mesh and switches to $44.370 \mathrm{kHz}$ for the coarse mesh. These two peak frequencies are associated with the shock screech and instabilities of underexpanded jets and will be discussed in Secs. IV.D and IV.E in detail. Here, they are mentioned insofar as to establish the independence of grid resolutions. Note that the amplitude of $f_{s}$ is higher than that of $f_{2 s}$ for the fine mesh but lower than that of $f_{2 s}$ for the coarse mesh. This behavior is mainly due to a shock screech frequency switching phenomenon, which will be discussed in Sec. IV.D as well. In addition, the visual area under the curve indicates the energy associated over those frequencies. From Fig. 5b, the spectrum curve for the fine mesh is on the top of the coarse mesh at high frequencies, which implies that more fluctuating energy associated with the turbulence motion is resolved by the fine mesh. Therefore, the result obtained with the fine mesh is shown hereafter.

Pope [53] proposed that, in an adaptive LES, the value of the turbulence-resolution tolerance $\varepsilon_{M}$ is set around 0.2 , which means that $80 \%$ of the turbulent kinetic energy should be resolved directly, whereas $20 \%$ needed to be modeled. The mean profiles of the total turbulent kinetic energy (TKE) and the SGS turbulent kinetic energy $k^{\text {sgs }}$ are plotted in Fig. 6a. The evolution of TKE is highly nonlinear, and there is no streamwise region where the exponential growth consistent with linear theory is sustained. On the other hand, the shocks modulate the TKE. These modulations are indicated by the dips before the shocks and a subsequent recovery of the TKE downstream of the shock, which agrees well with the measurements of instability wave growth for a supersonic rectangular jet done by Raman and Rice [54]. The ratio between the $k^{\text {sgs }}$ and TKE is slightly modulated by the shocks as well, which peaks at the first normal

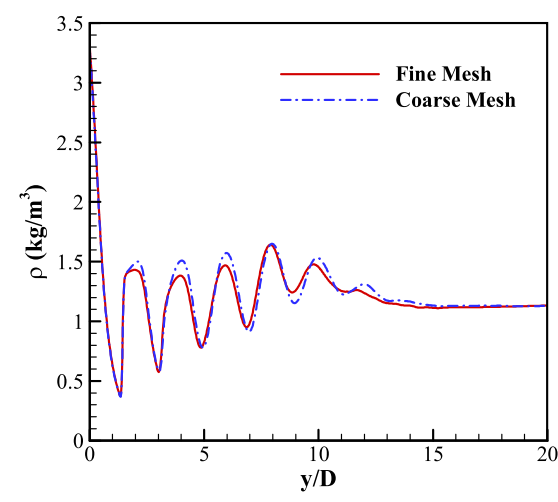

a)

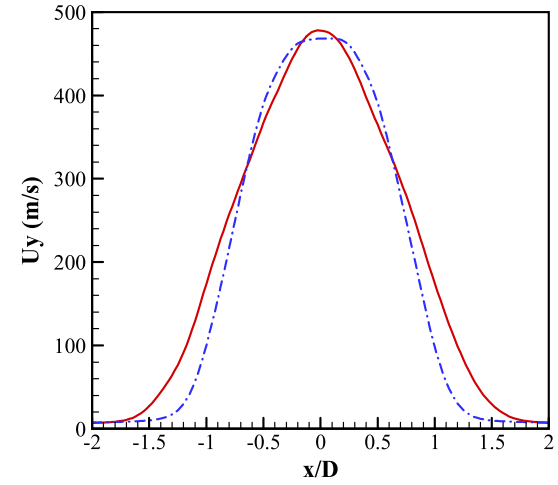

b)

Fig. 4 Grid independence analysis for NPR = 5.60: a) mean density along the underexpanded jet centerline, and b) radial profiles of the mean streamwise velocity at $y / D=10$.
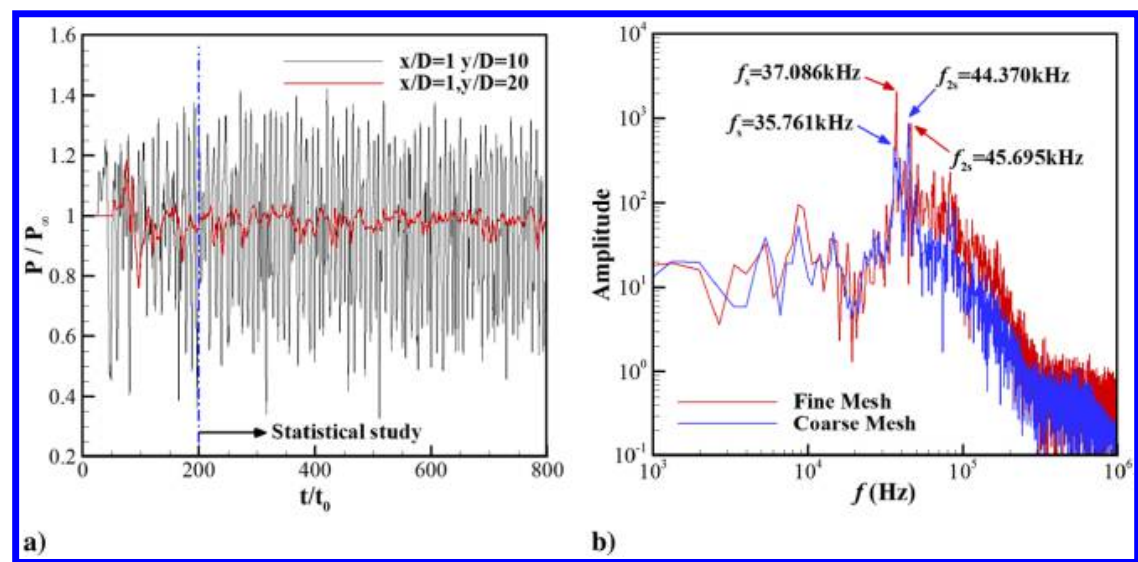

Fig 5 Representations of a) time history of instantaneous pressure based on the fine mesh, and b) spectrum of pressure fluctuation sampled at $x / D=1$, $y / D=6$, and $z / D=0$ for the two mesh resolutions. 


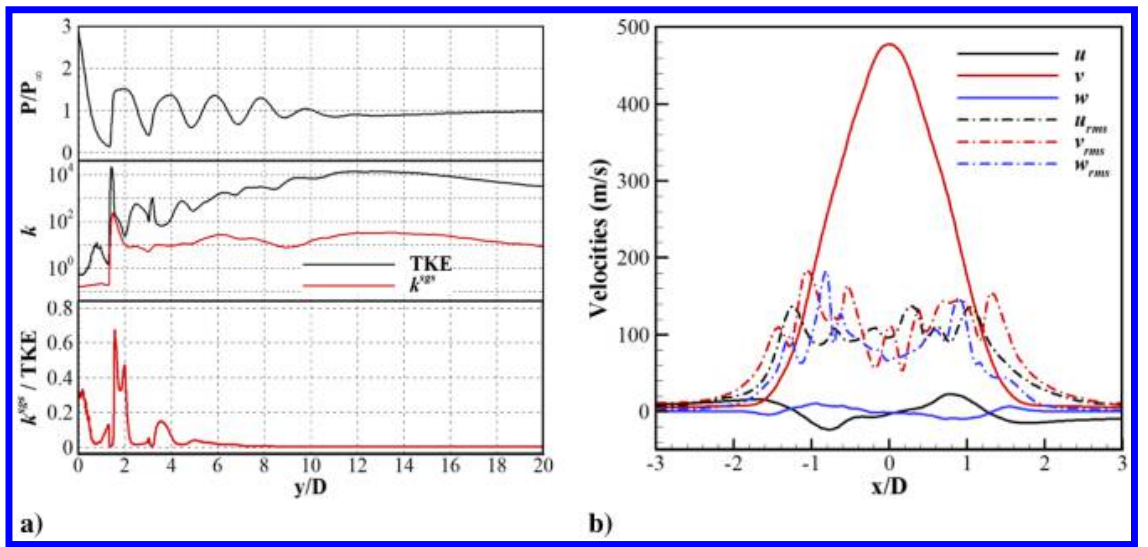

Fig 6 Representations of a) mean profiles of pressure, total TKE, SGS TKE $\left(k^{\mathrm{sgs}}\right)$, and the ratio between $k^{\text {sgs }}$ and TKE along the jet centerline; and b) radial profiles of the mean and fluctuating velocity components at $y / D=10$.

shock and decreases to 0.2 around the second normal shock. The radial profiles of the mean and fluctuating velocity components at $y / D=10$ are presented in Fig. $6 \mathrm{~b}$. The fluctuating velocities in the spanwise direction (i.e., $u_{\text {rms }}$ and $w_{\text {rms }}$ ) are on the same order with $v_{\text {rms }}$ but are one order larger than the mean velocities $\bar{u}$ and $\bar{w}$, which implies that the free shear-layer flows considered in this work are highly nonisotropic. This may be the reason why the ratio between the $k^{\text {sgs }}$ and TKE is much smaller than 0.2 after the second normal shock. It also implies that the grid resolution employed is high enough to resolve the turbulent properties of underexpanded jets.

\section{B. Time-Averaged Mach Disk}

Figure 7 shows the time-averaged contours of temperature, pressure, Mach number, and density gradient in the near field of the jet for NPR $=5.60$. As can be seen, the nitrogen jets are sped up to about sonic speed at the nozzle exit, where the temperature is $T_{1} \approx 300.0 \mathrm{~K}$, the pressure is $P_{1} \approx 0.3 \mathrm{MPa}$, and the Mach number is $M a \approx 1.0$. The main flow structures in the near nozzle region, including the Mach disk, triple point, reflected shock, and the slip lines, which have been confirmed by the previous experimental and numerical work [1-3,5-11,30,32], are well captured by the present LES shown in Fig. 7d. The triple point is the intersection of the intercepting shock, the Mach disk, the first reflected shock, and the slip line. The rapid expansion leads to a region of low temperature $\left(\bar{T}_{\min } \sim 100.0 \mathrm{~K}\right)$, low pressure, and high velocities $\left[\left(U_{y}\right)_{\max } \sim\right.$ $\left.700 \mathrm{~m} / \mathrm{s} \approx 2 U_{1},(M a)_{\max } \sim 3.0\right]$ in the vicinity of the Mach disk. Then, the flow is compressed strongly by the Mach disk, which increases the temperature, density, and pressure. The flow becomes subsonic $(M a \sim 0.2)$ again immediately after the Mach disk.

Yang [55] has measured the underexpanded nitrogen jets with a nozzle total pressure from 0.25 to $5.0 \mathrm{MPa}$ using schlieren photography, where the experimental data are represented by timeaveraged results over an exposure time of $0.6 \mathrm{~ms}$. Figure 8 compares the time-averaged density gradient obtained from LES and schlieren photography for the four simulated cases. Good agreements in the structures can be observed in the region $y / D<5$. In particular, the locations of the Mach disk as well as the first and the second reflected shock on the jet boundary are well captured by LES.

The Mach disk height $H_{m}$ and diameter $W_{m}$ predicted by LES are quantitatively summarized in Table $\underline{3}$. Ashkenas and Sherman [56] obtained an empirical formula for the Mach disk height $H_{m}$ for the range of NPR from 20 to 200 as

$$
\frac{H_{m}}{D}=C_{H} \cdot \sqrt{\frac{P_{0}}{P_{\infty}}}
$$

where $C_{H}$ is a constant of 0.67 . Ewan and Moodie [57] did experiments and observed a much smaller value of $C_{H} \approx \overline{0.55}$ for NPR $<10$ but with $C_{H}$ approaching 0.67 with increasing NPR. Franquet et al. [7] reviewed the progress in underexpanded jets and suggested a value of $C_{H}=0.645497$. The present LES produces a $C_{H}$ of $0.60,0.62,0.63$, and 0.64 for NPR $=5.60,7.47,9.34$, and 11.21, respectively, which increases slightly with NPR and is between the experimental data by Ewan and Moodie [57] and

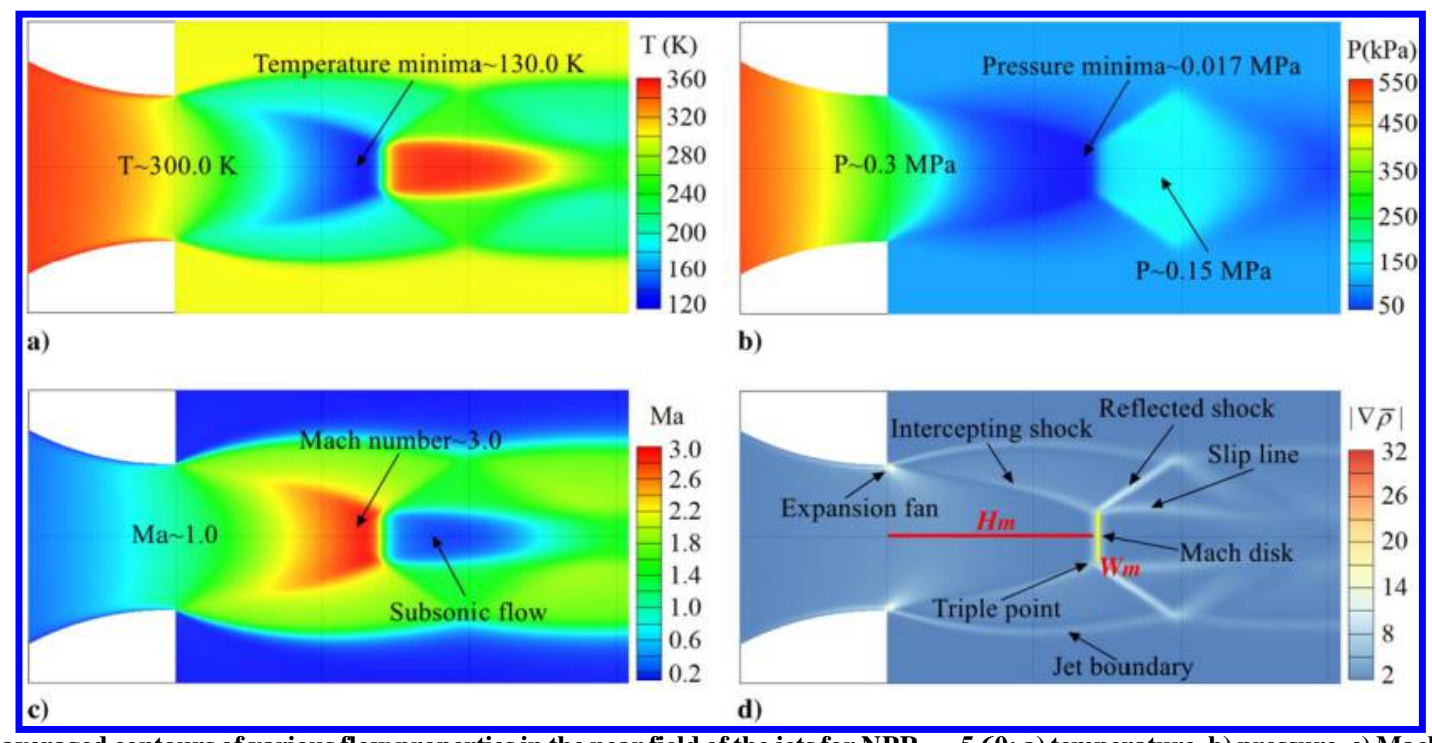

Fig 7 Time-averaged contours of various flow properties in the near field of the jets for NPR $=5.60:$ a) temperature, b) pressure, c) Mach number, and d) density gradient $(|\nabla \bar{\rho}|)$. 

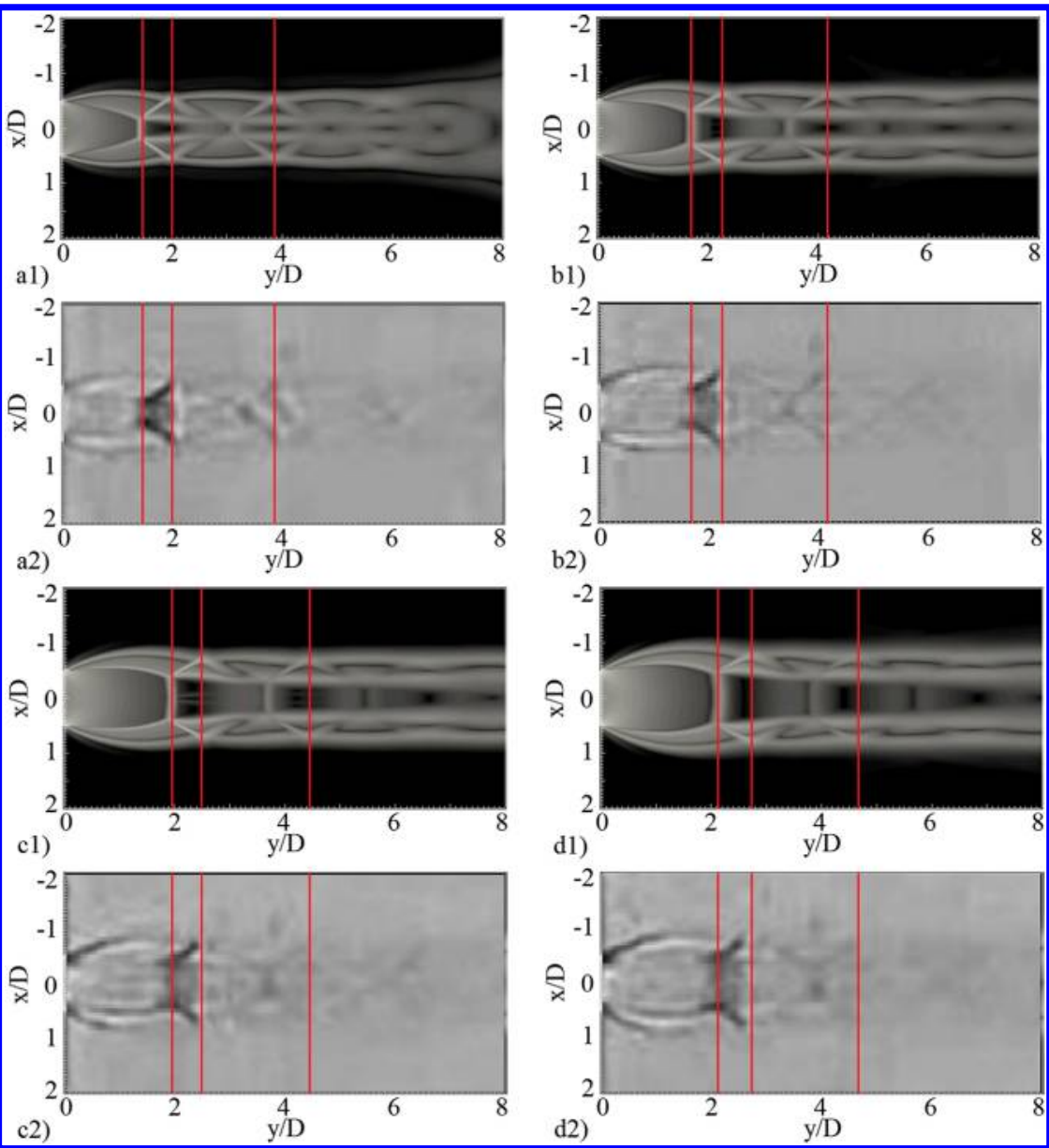

Fig. 8 Comparison of the time-averaged density gradient obtained from LES (a1, b1, c1, d1) and schlieren photography (a2, b2, c2, d2) [55]: a) $\mathrm{NPR}=5.60$, b) $\mathrm{NPR}=7.47$, c) $\mathrm{NPR}=9.34$, and d) $\mathrm{NPR}=11.21$.

Ashkenas and Sherman [56], yet close to the value proposed by Franquet et al. [7] and the $\overline{\mathrm{LESS}}$ prediction by La Vuorinen et al. [32].

\section{Results and Discussion}

\section{A. Turbulence Transition Processes}

Figure 9a shows the mean and fluctuating profiles of pressure along the underexpanded jet centerline at NPR of 5.60, which qualitatively characterizes the extent to which the instantaneous flowfield differs from the time-averaged one. The intensity of pressure fluctuations is relatively small in the near field and grows rapidly with increasing distance, until it peaks around $y / D=10$, from which a fully developed turbulent flow is established. The intensity of pressure fluctuations is also modulated periodically by the shocks and spreads toward to the radial direction. After $y / D=10$, the fluctuations decay slowly toward zero. It is also found that the temporal fluctuations mainly originate from within the shear layer, including both the inner (the slip line) and outer (the jet boundary) shear layers. The fluctuations in the inner shear layer grow earlier than those in the outer shear layer, whereas the intensity of pressure fluctuations in the outer shear layer is larger. The high-level fluctuations in the shear layer will result in higher shear-layer instability, which is believed to be one of the primary mechanisms that cause transition to turbulence in jet flows.

Figure $9 \mathrm{~b}$ shows the mean and fluctuations of vorticity magnitude, which exhibit similar overall qualitative characteristics as the pressure profiles (i.e., the intensity of vorticity fluctuations grows rapidly with increasing axial distance), but significant quantitative differences are evident as well. In particular, the overall ratio of instantaneous vorticity fluctuations to the mean is significantly higher than those of pressure throughout the jet. In fact, the instantaneous vorticity values in several profiles are almost an order of magnitude larger than the mean values, which is consistent with the general features of turbulent flows characterized by intense vorticity fluctuations [58].

Based on the similar detailed analysis on the flow characteristic for the four cases associated with the jet penetration and maximum width to be discussed next, the turbulent transition processes of highly

Table 3 Comparison of the Mach disk height and diameter

\begin{tabular}{|c|c|c|c|c|c|c|}
\hline Author & Technique & $D, \mathrm{~mm}$ & $P_{0} / P_{\infty}$ & $H_{m} / D$ & $W_{m} / D$ & $C_{H}$ \\
\hline Ashkenas and Sherman [56] & Experiment & -- & $20 \sim 200$ & -- & -- & 0.67 \\
\hline Ewan and Moodie [57] & Experiment & - - & $\sim 10$ & -- & -- & 0.55 \\
\hline Franquet et al. [7] & Review & -- & -- & -- & -- & 0.64549 \\
\hline La Vuorinen et al. [32] & LES & 1.4 & 5.50 & 1.43 & 0.36 & 0.61 \\
\hline La Vuorinen et al. $[\overline{32}]$ & LES & 1.4 & 7.50 & 1.72 & 0.55 & 0.63 \\
\hline Present work & LES & 2.0 & 5.60 & 1.43 & 0.38 & 0.60 \\
\hline Present work & LES & 2.0 & 7.47 & 1.70 & 0.54 & 0.62 \\
\hline Present work & LES & 2.0 & 9.34 & 1.93 & 0.76 & 0.63 \\
\hline Present work & LES & 2.0 & 11.21 & 2.14 & 0.90 & 0.64 \\
\hline
\end{tabular}



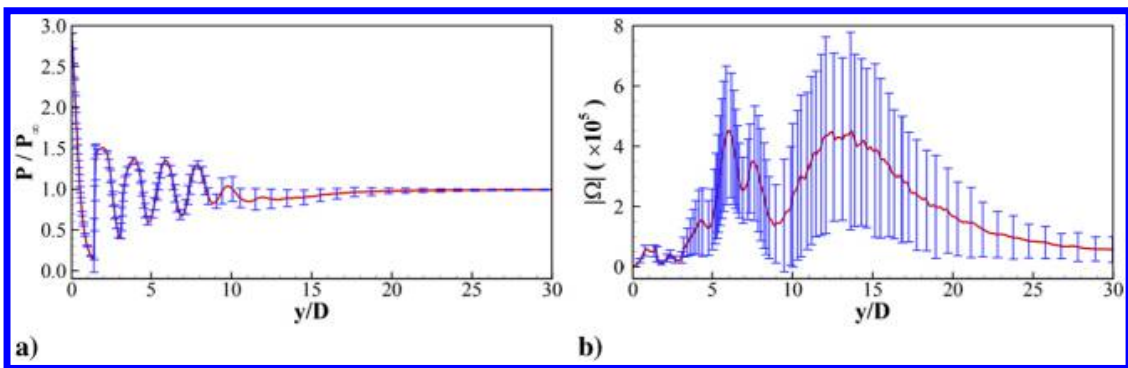

Fig. 9 Mean profiles of a) pressure, and b) vorticity magnitude, along the jet centerline for NPR $=5.60$. Vorticity magnitude is defined as $|\Omega|=\left(\Omega_{x}^{2}+\Omega_{y}^{2}+\Omega_{z}^{2}\right)^{1 / 2}$ and $\Omega=\nabla \times u$. The error bars represent the rms of the fluctuating values.

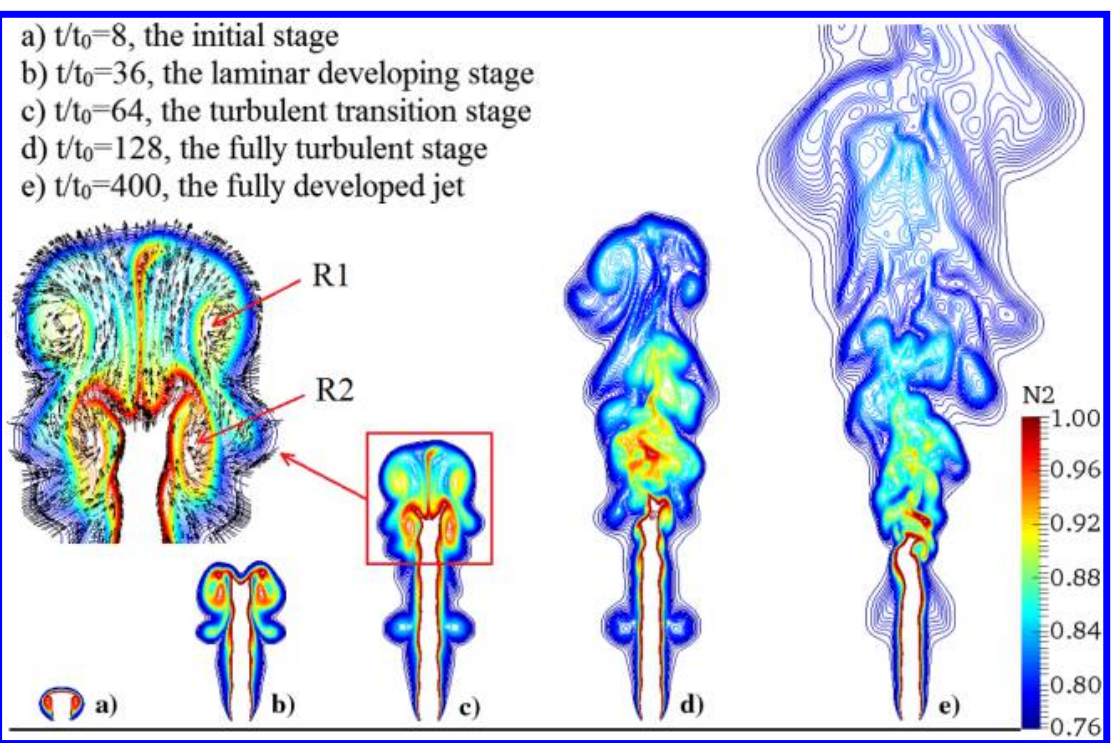

Fig. 10 Instantaneous snapshots of contour lines of nitrogen mass fraction for NPR $=5.60$. The arrows in Fig. 10c represent the local velocity vector. R1 and $\mathrm{R} 2$ represent the first and second recirculation zones.

underexpanded sonic jets can be defined as four different flow stages: 1) the initial stage, 2) the laminar developing stage, 3) the turbulent transition stage, and 4) the fully turbulent stage, which is indicated in Figs. $10 \mathrm{a}-10 \mathrm{~d}$ by the nitrogen mass fraction and in Figs. $11 \mathrm{a}-11 \mathrm{~d}$ by the $Q$-criterion [58]:

$$
Q=\frac{1}{2}\left(\Omega_{i j} \Omega_{i j}-S_{i j} S_{i j}\right)
$$

where $S_{i j}$ and $\Omega_{i j}$ represent the strain and rotation tensor, respectively.

At the initial stage, the nitrogen jets burst out of the nozzle and are blocked by the quiescent ambient air, forming the first recirculation zone. At the same time, a tip vortex ring, which is also visible in subsonic jets $[59,60]$, rises and grows while convecting downstream. The first recirculation zone keeps increasing until reflecting back at the jet's core, resulting in an undulating vortex ring outside of the second normal shock. Meanwhile, more vortex rings form and move parallel downstream. There are pairs of streamwise vortices of small magnitude formed after the tip vortex ring, but they are symmetric with respect to the streamwise direction (the $y$ axis). Therefore, the flow is believed to be still laminar, and this stage is called the laminar developing stage. As time goes on, a second recirculation zone is formed gradually, and vortex rings of small magnitudes break down. The flow becomes unstable, while the tip vortex ring and the streamwise vorticity lose their symmetry; the coannular shear layer and the undulating vortex ring get distorted slightly due to the presence of Kelvin-Helmholtz instabilities, resulting in the turbulent transition stage. Then, a third recirculation zone is formed that quickly breaks into a large number of small recirculation zones, and the tip vortex ring also breaks down and loses its symmetry. At the same time, most of the vortex rings in the near field of the jet are distorted, the undulating vortex ring outside of the third coannular shear layer disappears gradually, and large-scale $\Omega$ shape vortices are visible along the shear layer, indicating the fully developed stage. These turbulent characteristics have also been identified in previous numerical modeling of supersonic underexpanded jets [32,52,61]. The fully developed jet is shown in Figs. 10e and 11e. It can be seen that the large-scale $\Omega$ shape vortices along the shear layer are rotating by themselves and nesting mutually due to three-dimensional KelvinHelmholtz (K-H) instabilities but also moving in the downstream direction; accordingly, complex chains of vortex rings occur.

It is worth noticing that there are interim stages between two adjacent flow stages. The interim stages last for a short time and provide transition in structure from one stage to the next flow stage. In addition, the LES shows that the second flow stage shortens with increasing NPR because the turbulent transition process is faster for higher NPR, implying higher Reynolds numbers. Figures 12 and 13 show that the weak turbulence takes place at about $t / t_{0}=40 \overline{\text { for }}$ $\mathrm{NPR}=9.34$, which is earlier than for the NPR $=5.6$ case, where it takes place at $t / t_{0}=64$. The delimitation between the laminar developing stage and the turbulent transition stage is not clear for highNPR cases. This is mainly because the number of shock cells and the length of the jet core increase with NPR, and the second recirculation zone subsequent to the first one cannot be identified as clearly as for the low-NPR cases. On the other hand, the delimitation between the turbulent transition stage and the fully turbulent stage can be clearly defined for all cases by the interim stage, which is characterized by the breakdown of recirculation zones, the loss of the flow symmetry, the distortion of vortex rings in the near field (the coannular shear layer) of the jets, and the generation of streamwise vortices. 

a) $t / t_{0}=8$, the initial stage
b) $t / t_{0}=36$, the laminar developing stage
c) $t / t_{0}=64$, the turbulent transition stage
d) $t / t_{0}=128$, the fully turbulent stage
e) $t / t_{0}=400$, the fully developed jet
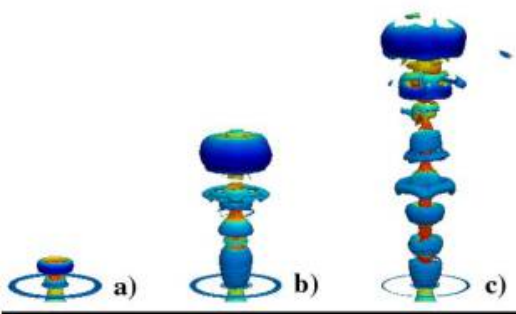

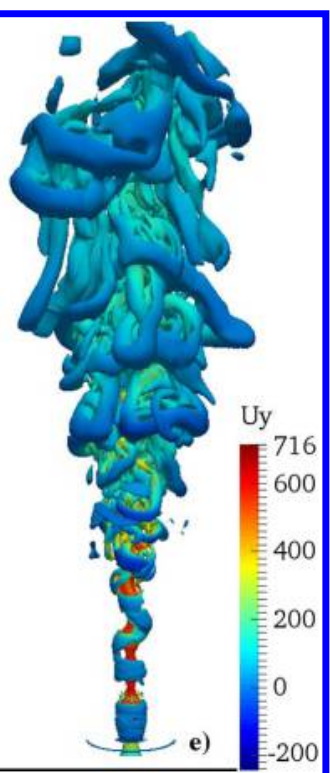

Fig. 11 Three-dimensional isosurfaces of the $Q$-criterion $\left(Q=10^{8} \mathrm{~s}^{-2}\right)$ colored by streamwise velocities for NPR $=5.60$. The corresponding times for Figs. 11a-11e are the same with Fig. 10.

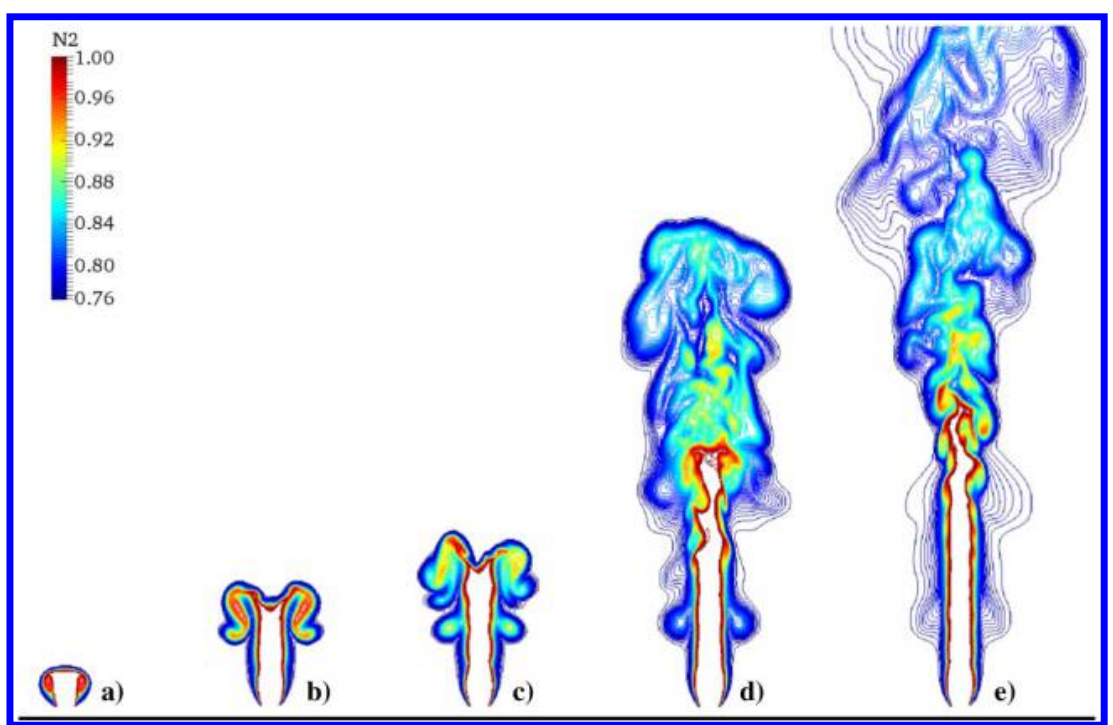

Fig. 12 Instantaneous snapshots of contour lines of nitrogen mass fraction for NPR $=9.34:$ a) $\left.t / t_{0}=8, \mathrm{~b}\right) t / t_{0}=28$, c) $\left.t / t_{0}=40, \mathrm{~d}\right) t / t_{0}=128$, and e) $t / t_{0}=400$. Figures 12a-12e represent the same flow stages as Fig. 10. The turbulence transition process is faster at increased NPR.

\section{B. Jet Penetration and Maximum Width}

The time-dependent jet penetration $z(t)$ and maximum width $W$, which determine the volumetric rate of the jets and are closely related to the overall mixing and entrainment, are two important overall parameters to characterize the flow evolution characteristics. In contrast to previous studies [8], where a symmetric fuel concentration profile was introduced at the midline plane in a RANS simulation and hence defining the jet penetration, in the present study the jet penetration and maximum width are defined according to the outer limit of nitrogen mass fraction on the midline plane to account for the asymmetry effects. In other words, the jet penetration $z(t)$ is defined as the maximum axial position that is not always on the centerline, and the jet maximum width is defined as the maximum span in the radial direction. In this definition, the maximum width of the jets may be slightly overestimated because the maximum and minimum radial positions do not always occur at the same axial position, as seen in Figs. 10 and 12 .

Ouellette [62] investigated the penetration rates in the far downstream of underexpanded jets using schlieren photography at $R e \sim 5.0 \times 10^{5}$ and found that the penetration of the fuel jets obeys a linear dependency on the square root of time. Ouellette and Hill [ $[\mathrm{g}]$ and Hill and Ouellette [63] derived a formula for the penetration of underexpanded sonic jets for distances greater than 20 nozzle diameters and jet Reynolds number greater than $3 \times 10^{4}$ as

$$
\frac{z(t)}{\left(\dot{M}_{n} / \rho_{\infty}\right)^{1 / 4} t^{1 / 2}}=\Gamma
$$

where $z(t)$ is the jet penetration, $\dot{M}_{n}$ is the momentum injection rate, $t$ is the time from the beginning of injection, $\Gamma$ is a constant equal to $3.0 \pm 0.1$ for turbulent jets issued from round nozzles. Note that Eq. (16) can be transformed directly to the following form [8]:

$$
\frac{z(t)}{d_{\mathrm{eq}}}=\Gamma\left(\frac{\pi}{4}\right)^{1 / 4}\left(\frac{t U_{1}}{d_{\mathrm{eq}}}\right)^{1 / 2}
$$

where $d_{\mathrm{eq}}$ is the equivalent diameter $d_{\mathrm{eq}}=D\left(\rho_{1} / \rho_{\infty}\right)^{1 / 2}$, and $\rho_{1} / \rho_{\infty}$ is the nozzle density ratio (NDR).

Figure 14a compares the predicted jet penetration and the measured data. As can be seen, the jet penetration predicted by LES is in good agreement with the experimental data in spite of differences in NDR. However, there are some differences to be noticed. First, the jet penetration in the initial stage, which is rarely discussed in the 


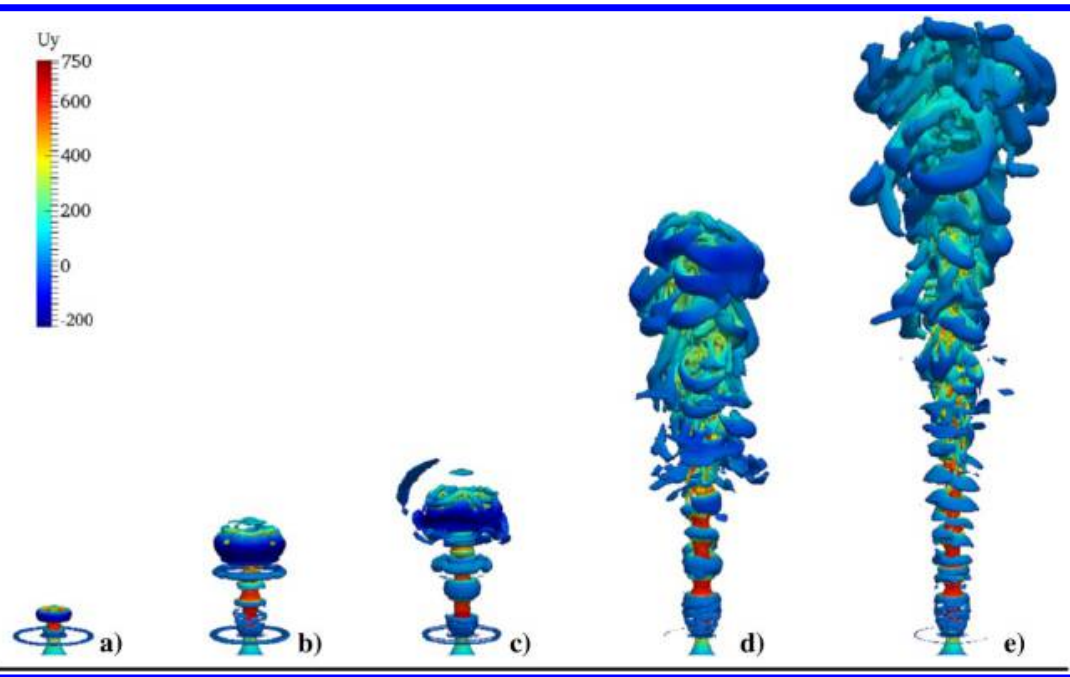

Fig. 13 Three-dimensional isosurfaces of the $Q$-criterion $\left(Q=10^{8} \mathrm{~s}^{-2}\right)$ colored by streamwise velocities for NPR $=9.34$. The corresponding times for Figs. 13a-13e are the same as Fig. 12.

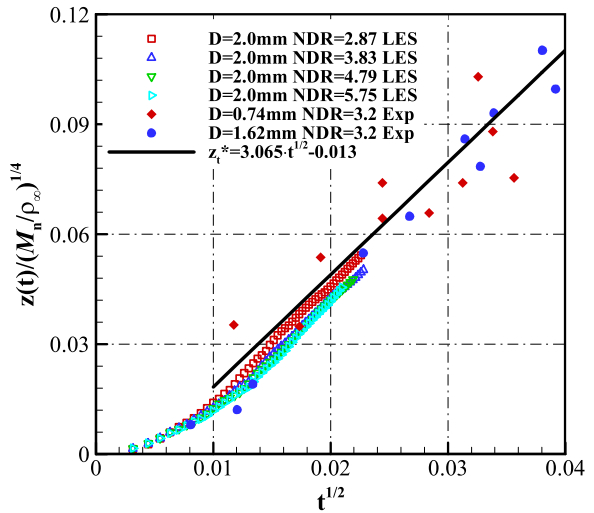

a)

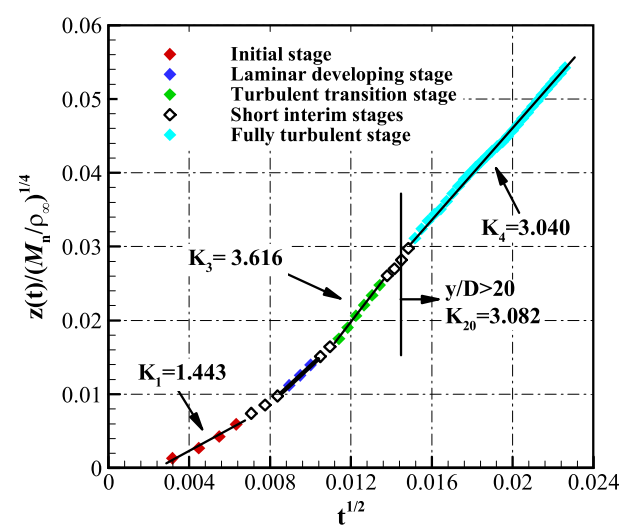

b)

Fig. 14 Representations of a) the comparison between the predicted penetration rates of jets and the measured data by Miyake as quoted in [8], and b) nondimensional penetration rates in different flow stages for NPR $=5.60$ and NDR $=2.87$ case.

previous studies, differs greatly with that in the fully developed stage. In addition, the slope of jet penetration after $20 D$ from the nozzle exit, which is suggested to be a constant of $3.0 \pm 0.1[\underline{8}, 63]$, seems to vary with NDR. To investigate the jet penetration rate at different flow stages quantitatively, a linear fit similar to Eqs. (16) and (17) is implemented for different flow stages as

$$
z_{t}^{*}=K \cdot t^{1 / 2}-B
$$

where $z_{t}^{*}$ is the nondimensional jet penetration $z(t) /\left(\dot{M}_{n} / \rho_{\infty}\right)^{1 / 4}$, and $K$ is the slope. Figure $14 \mathrm{~b}$ shows the linear fit for the NPR $=5.60$ and the NDR $=2.87$ case. Table 4 summarizes the computed results, where $K 1, K 3, K 4, K 20$, and $K_{\text {overall }}^{-}$are the slopes calculated based on the data in the first flow stage, data in the third flow stage, data in the fourth flow stage, data after 20 diameters from the nozzle exit, and all the data points. The second flow stage is relatively short compared with other flow stages; thus, the linear fit process is not implemented.

From Table 4, the current LESs show that the slope of nondimensional penetration after $20 D$ from the nozzle exit (i.e., $K 20$ ) for the NDR $=2.87$ and 3.83 cases is around 3.0, which is close to the measurements by Miyake (as quoted in [8] ) under a similar NDR of 3.2. However, the slope $K 20$ increases when NDR keeps increasing. This trend is also confirmed by the experimental and RANS results by Ouellette and Hill [8]. Thus, the jet penetration coefficient $K 20$ is believed to be a function of NDR rather than a constant, and its dependency on NDR needs to be further resolved by measurements and simulations for a wider range of NDR. The location where the fully developed turbulence is established is around $y / D=20$ for $\mathrm{NDR}=2.87$ and then decreases gradually with increasing NDR because the increased Reynolds number speeds up

Table 4 Jet penetration constants in different flow stages for different NDR

\begin{tabular}{|c|c|c|c|c|c|c|c|c|c|c|}
\hline Author & Technique & $D, \mathrm{~mm}$ & NDR & $K_{\text {overall }}$ & $K 1$ & $K 3$ & $K 4$ & $K 20$ & $\Delta(K 20), \%$ & $\Pi_{40}$ \\
\hline Hill and Ouellette [63] & Theory & -- & -- & -- & -- & -- & -- & $3.0 \pm 0.1$ & \pm 3.33 & - \\
\hline Miyake (quoted in [주) & Experiment & 0.74 & 3.2 & -- & -- & -- & -- & 3.065 & 2.17 & -- \\
\hline Miyake (quoted in $[\overline{8}]$ ) & Experiment & 1.62 & 3.2 & -- & -- & -- & -- & 3.065 & 2.17 & - - \\
\hline Ouellette and Hill [] & Experiment /RANS & 0.5 & $0.63 \sim 2.01$ & -- & -- & -- & -- & 2.65 & -11.67 & - \\
\hline Present work & LES & 2.0 & 2.87 & 2.991 & 1.443 & 3.616 & 3.040 & 3.082 & 2.73 & 0.27 \\
\hline Present work & LES & 2.0 & 3.83 & 2.739 & 1.399 & 2.513 & 3.039 & 2.978 & -0.73 & 0.31 \\
\hline Present work & LES & 2.0 & 4.79 & 2.697 & 1.348 & 2.324 & 3.212 & 3.238 & 7.93 & 0.35 \\
\hline Present work & LES & 2.0 & 5.75 & 2.653 & 1.292 & 2.258 & 3.404 & 3.458 & 15.27 & 0.36 \\
\hline
\end{tabular}


the turbulent transition. Therefore, $K 4$, which is the jet penetration coefficient in the fully turbulent stage and corresponds to the jet downstream that may not be included by the original $K 20$ definition, is proposed to describe the jet penetration behaviors in the downstream of the highly underexpanded jets. For example, $K 4$ characterizes the jet penetration from as early as $15 D$ for NDR $=4.79 . K 1$ is smaller than $K 3$ and $K 4$ for all four simulations and decreases slightly with increasing NDR. $K 3$ exhibits the same overall qualitative characteristics as $K 1$ but is larger than $K 1$ and smaller than $K 4$ except for NDR $=2.87$. The low $K 1$ and $K 3$ values can be explained by the observation that the jet propagates downstream much slower in the initial stages and accelerates gradually as turbulent transition occurs, accelerating more remarkably for higher NDR. Last, remarkable differences exist among $K 1, K 3, K 4, K 20$, and $K_{\text {overall }}$ for all four cases, which implies that the jet penetration behavior of highly underexpanded sonic jets cannot be characterized solely by $K 20$ or $K 4$ and remain to be further investigated in detail.

Rizk [64] measured the penetration rate of incompressible jets and found that the jets in the downstream reach a self-similarity, which can be expressed by a constant ratio of the jet maximum width $W$ to jet penetration $z(t)$ defined as follows [64]:

$$
W / z(t)=\Pi
$$

where $\Pi$ is a constant whose value is $0.25 \pm 0.05$. Diez et al. [65] measured unsteady round buoyant turbulent plumes and thermals jets for Reynolds numbers ranging from 4000 to 11,000 , and their photographs showed that $W / z(t)$ decreases rapidly with increasing streamwise distance in the near field $(y / D<20)$ and then reaches a constant 0.32 for starting plume jets and 0.38 for thermals jets after $30 \sim 40 D$ from the nozzle exit until far downstream $(y / D=120)$.

Figure 15a shows the nondimensional jet maximum width for different $\overline{\mathrm{NDR}}$ obtained by LES. The overall trend is the same as those of turbulent plumes and thermals jets measured experimentally by Diez et al. [65]. At the early beginning of the jets, $W / z(t)$ is larger than 1 , then decreases rapidly with streamwise distance in the near field $(y / D<20)$, and approaches a constant in the far downstream. Limited by the computational length in the current study, the values of $W / z(t)$ at the maximum axial distance $y / D=40$ (i.e., $\Pi_{40}$ ) are shown in Table 4 . Although the jet maximum width is slightly overpredicted, the nondimensional jet maximum width $\Pi_{40}$ predicted by LES is between the measurements by Rizk [64] and Diez et al. [65]. Note that the jet flow has become subsonic already at $y / D=40$. From the overall trend, the nondimensional jet maximum width of the highly underexpanded sonic jets tends to reach a constant around 3.0 in the far downstream.

Figure $15 \mathrm{a}$ also shows some special zigzag structures characterized by sudden jumps of $W / z(t)$, which are generally

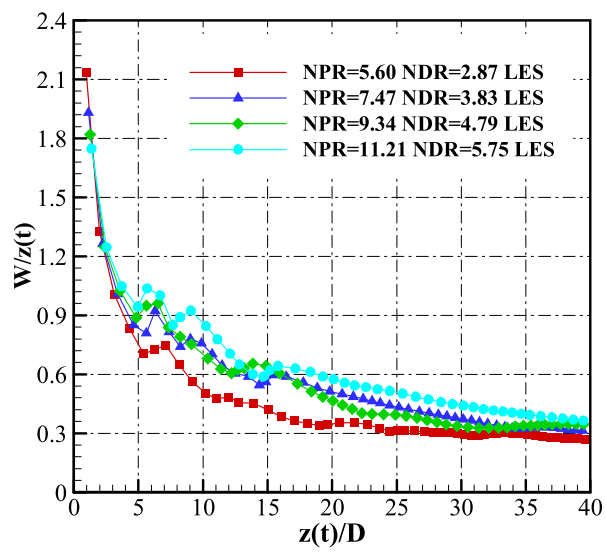

a)

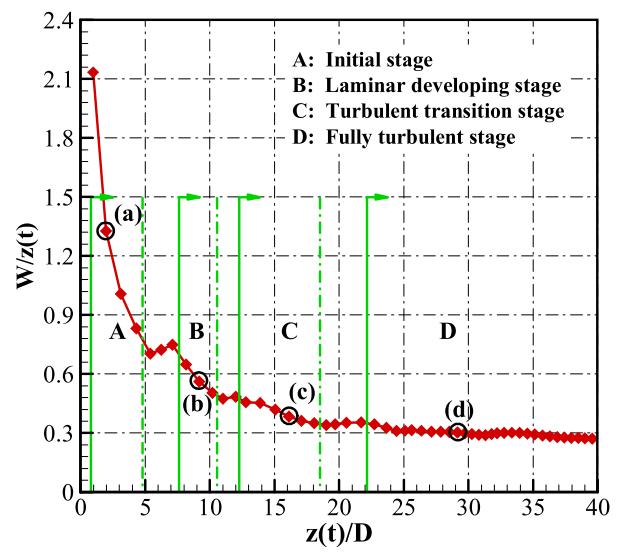

b)

Fig. 15 Representations of a) nondimensional jet maximum width of underexpanded sonic jets as functions for, and b) the flow stages divided based on jet maximum width for the NPR $=\mathbf{5 . 6 0}$ and NDR $=2.87$ case. The zones between two adjacent solid and dash-dotted lines are different flow stages, whereas the zones between two adjacent dash-dotted and solid lines are interim stages. The points a-d) circled with black solid line correspond to Figs. 10a-10d and 11a-11d. inaccessible by experimental technique. There are usually three zigzag structures, and the time that these zigzag structures experience is relatively short. From the preceding analysis on the turbulent transition processes, these three zigzag structures correspond to the three interim stages and are related to the recirculation zone productions. For example, the first recirculation zone keeps increasing after the initial stage and leads to a sudden jump for the jet maximum width, resulting in the first zigzag structure. As the time goes on, a second recirculation zone forms, and the weak turbulence takes place as well, which leads to the generation of another set of zigzag structure. The formation of the third zigzag structure is similar to the first two and is accompanied by the generation of the third recirculation zone. At last, different flow stages can be obtained based on jet maximum width profile, and Fig. 15b shows an example for $\mathrm{NPR}=5.60$. Note that the discussions on turbulence transition and jet penetration are based on a linear initialization of pressure and temperature inside the nozzle indicated by Fig. $\underline{2}$, and there could be some differences compared to the flow evolution of jets entering the ambient air under realistic conditions, mainly at the initial stage.

\section{Vorticity Analysis}

Figure 16 shows instantaneous snapshots of density gradient magnitude in cross-section planes for different flow stages. It can be seen that the vortex rings move parallel in the downstream direction in the laminar developing stage and get distorted gradually in the turbulent transition stage such as at $y / D=10$, until the streamwise vortices of large magnitude generate in the fully developed stage. This provides new evidence for the conclusions discussed previously that the turbulent transition processes are characterized by the distortion of the vortex rings and generation of streamwise vortices. This turbulent transition mechanism has been also observed in square [66] and elliptical [67] jets. It can also be observed from Fig. 16 that the vortex rings in the near field (for example, at $y / D=2$ ) are not distorted in the laminar developing stage but get distorted gradually as the turbulent transition takes place, implying that the instabilities in the shear layer have been transported upstream as time evolves.

Analysis of the driving forces to distort the vortex rings and form the streamwise vortices is conducive to understand the turbulent transition mechanisms. Vorticity evolution is ruled by the compressible vorticity transport equation defined as

$$
\begin{aligned}
& \underbrace{\frac{\partial \boldsymbol{\omega}}{\partial t}}_{I}=\underbrace{-(\boldsymbol{v} \cdot \nabla) \boldsymbol{\omega}}_{\text {conv }}+\underbrace{(\boldsymbol{\omega} \cdot \nabla) \boldsymbol{v}}_{\text {VS }} \underbrace{-\boldsymbol{\omega}(\nabla \cdot \boldsymbol{v})}_{\text {dila }}+\underbrace{\frac{1}{\rho^{2}} \nabla \rho \times \nabla p}_{\text {baro }} \\
& \quad+\underbrace{\nu \nabla^{2} \boldsymbol{\omega}}_{\text {diff }}
\end{aligned}
$$




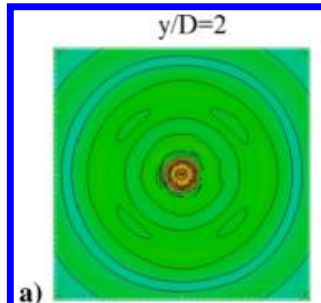

a)
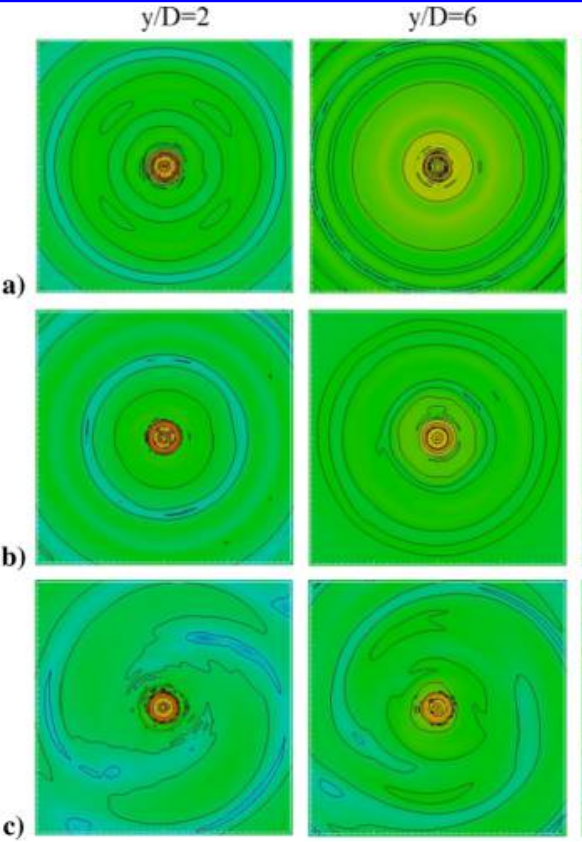
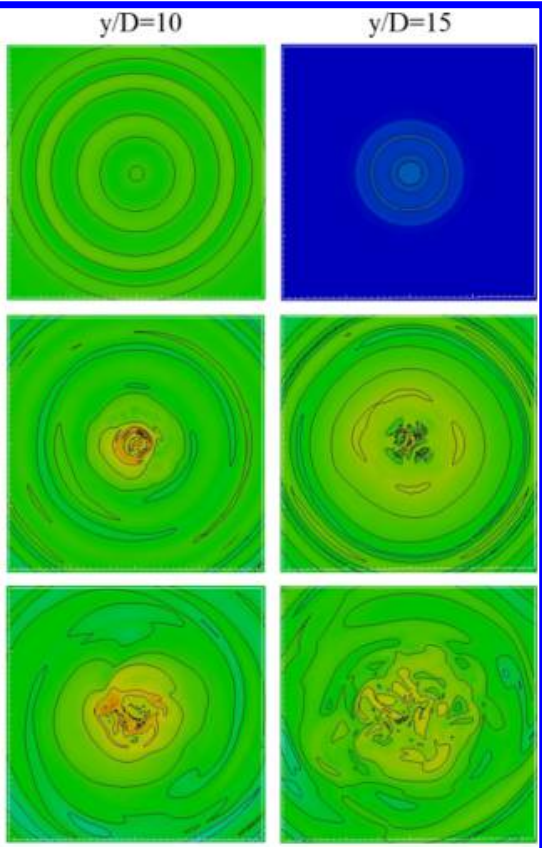

Fig. 16 Instantaneous snapshots of density gradient magnitude on different cross-section planes at different flow stages for NPR $=5.60:$ a) $t / t_{0}=36$, the laminar developing stage; b) $t / t_{0}=64$, the turbulent transition stage; and c) $t / t_{0}=400$, the fully developed jet.

where $I$, conv, VS, dila, baro, and diff are the inertial, convective, vortex stretching, dilatational, baroclinic, and diffusive terms, respectively. It can been seen that the convective, dilatational, and diffusive terms only change the magnitude of the vorticity, whereas the vortex stretching and baroclinic terms not only change the magnitude but also change the direction of the vorticity. Therefore, it is the vortex stretching and baroclinic terms that should be responsible for the distortion of vortex rings and the generation of streamwise vortices.

Figure 17 shows the instantaneous contours and Fig. 18 qualitatively presents the instantaneous profiles of magnitude of the convective, vortex stretching, dilatational, and baroclinic terms for $\mathrm{NPR}=5.60$. The four terms have approximately the same order of magnitude. This means that the dilatational and baroclinic terms, which are generally negligible in incompressible flows, are critical and play a key role in current underexpanded jet flows. The vorticity transport is not exclusively driven by vortex stretching but also by the compressibility and baroclinic effects. These observations are consistent with the previous nondimensional analysis [68] on supersonic internal flows as well as the LES modeling [69] of supersonic hydrogen/air combustion. Figures 17 and 18 also indicate that the baroclinic term, which is the coupling between density and pressure gradients, peaks mostly in the near field, especially at the triple points and the locations where reflected shocks intersect with the jet boundary. These peak locations correspond well to the shock structures reported in Fig. 8a (i.e., discontinuous regions with high density and pressure gradients). The dilatational term has a similar overall trend as for the distribution of the baroclinic term. However, the vortex stretching term mainly peaks along the outer and inner shear layers, where most vortices are generated. It is also observed

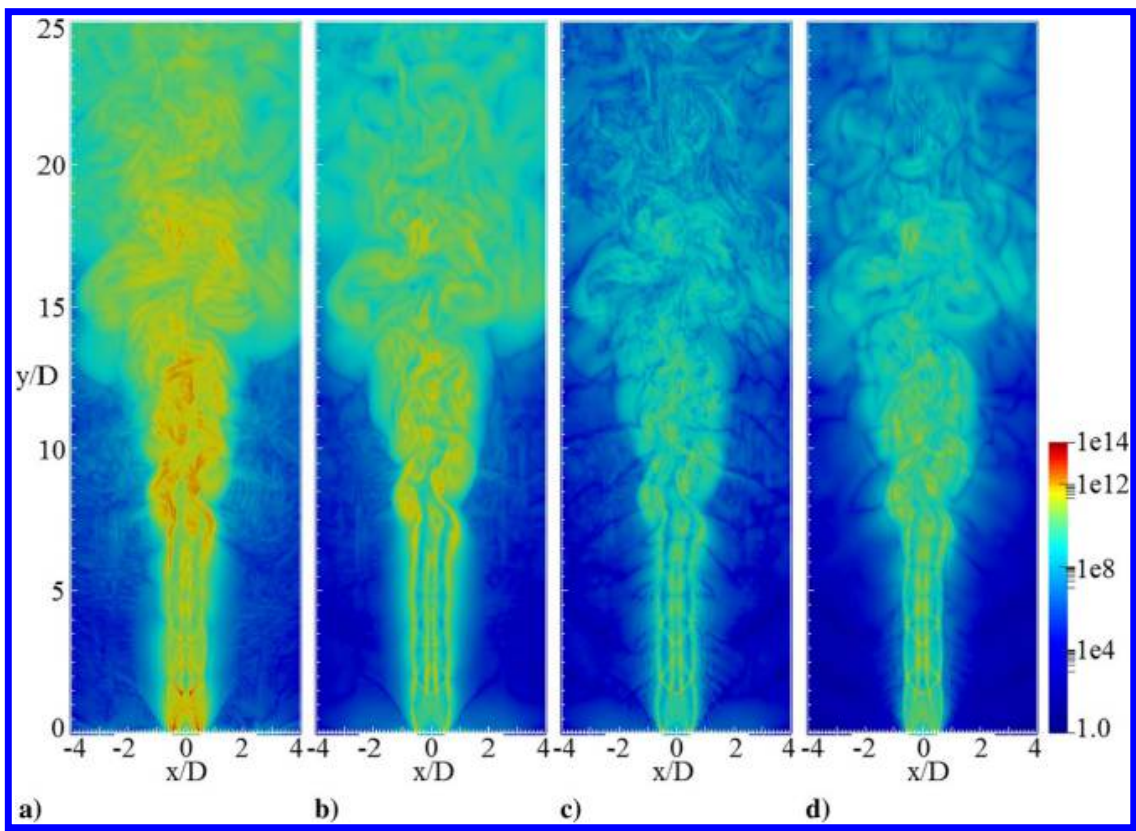

Fig. 17 Instantaneous snapshots of magnitude of the a) convective, b) vortex stretching, c) dilatational, and d) baroclinic terms on midline plane for highly underexpanded sonic jet of NPR $=\mathbf{5 . 6 0}$. 


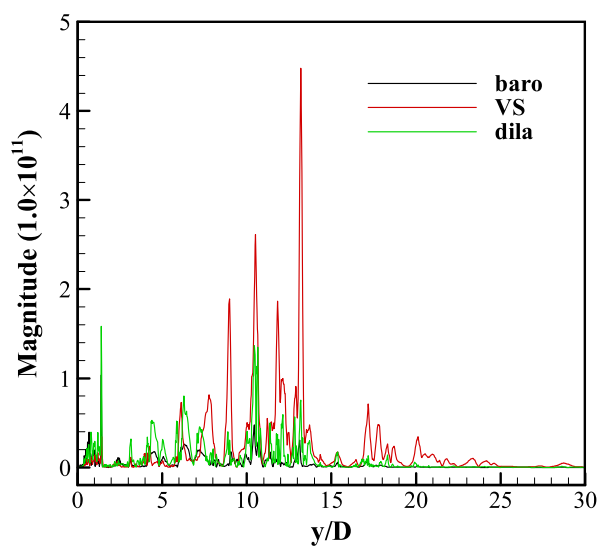

a)

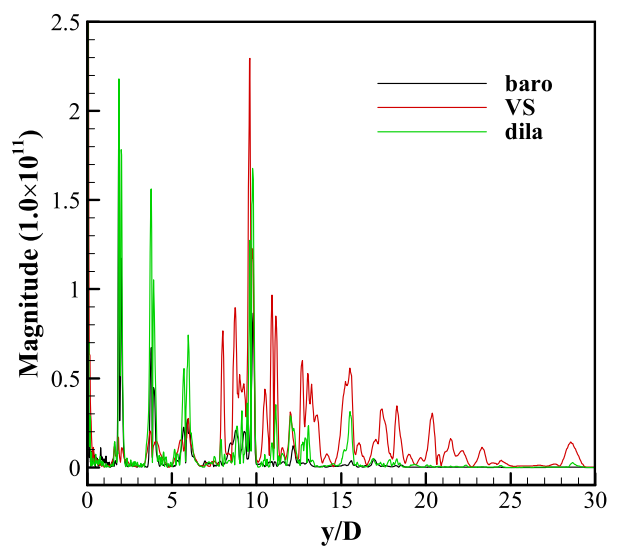

b)

Fig. 18 Instantaneous profiles of magnitude of the baroclinic ("baro"), vortex stretching ("VS"), and the dilatational ("dila") terms for NPR = 5.60 along a) jet centerline, and b) jet shear layer.

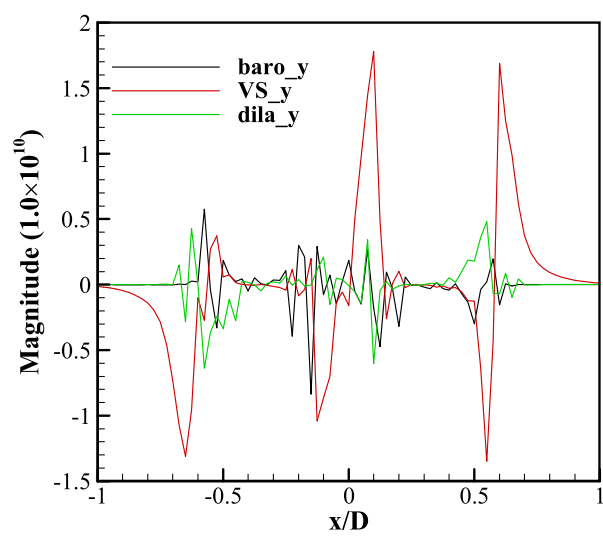

a)

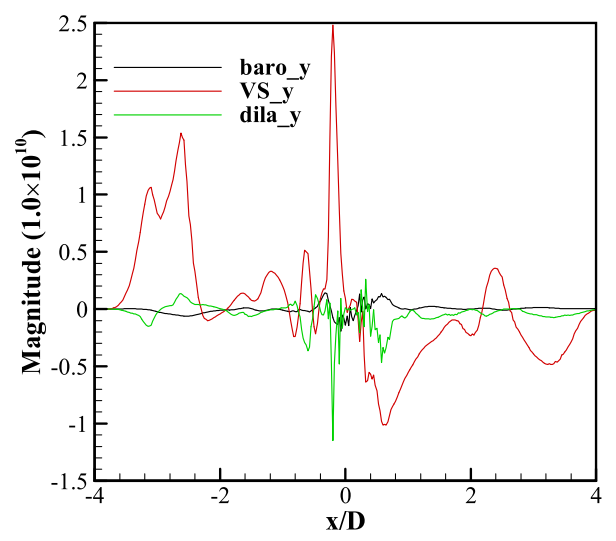

b)

Fig. 19 Radial profiles of the $y$ component of the baroclinic ("baro"), vortex stretching ("VS"), and the dilatational ("dila") terms for the fully developed jet at NPR of 5.60: a) $y / D=4$, and b) $y / D=15$.

from Fig. 18 that the magnitude of the baroclinic and dilatational terms is generally larger than the vortex stretching term in the near field of the jets $(y / D<7)$ because of quasi-periodic shock structures. However, the magnitude of the vortex stretching term is much larger in the downstream $(y / D>8)$ from where the large-scale turbulent vortices are periodically generated.

As stated previously, the transition to turbulence for highly underexpanded jets is associated with the distortion of vortex rings in the near field and the generation of streamwise vortices. Thus, the $y$ component of different terms in the vorticity transport equation need to be considered specially. Figure 19 shows the radial profiles of the $y$ component of the baroclinic, vortex stretching, and the dilatational terms at $y / D=4$ and $y / D=15$. Contrary to the observation from Fig. 18 that $\mid$ baro $\mid$ and $\mid$ dila $\mid$ are larger than $|\mathrm{VS}|$ in the near field of the jets, it is found that VS_y is much larger than baro_y and dila_y at $y / D=4$. This implies that the vortex stretching term plays the leading role in the distortion of vortex rings in the near field. Although the baroclinic term is involved, its effect is not as large as expected. On the other hand, VS_y, as expected, is dominant in the far downstream (see, for example, $y / D=15$ ) because of the fully developed subsonic turbulent flows.

\section{Dominant Instability Modes}

The time histories of the pressure signals measured near the shear layer from $t / t_{0}=400$ to $t / t_{0}=600$ are depicted in Fig. 20. The fluctuations at the two locations seem to be correlated and in phase opposition for NPR $=5.60$. As for the higher NPR of 9.34, the relation between the two pressure signals is much more complex, opposite or consistent randomly in phase. To reveal the screech instability modes in an in-depth way, the cross spectrum and relative phase between the two pressure probes on either side of the jets at different streamwise positions of $y / D=2,4,6,8$, and 10 are
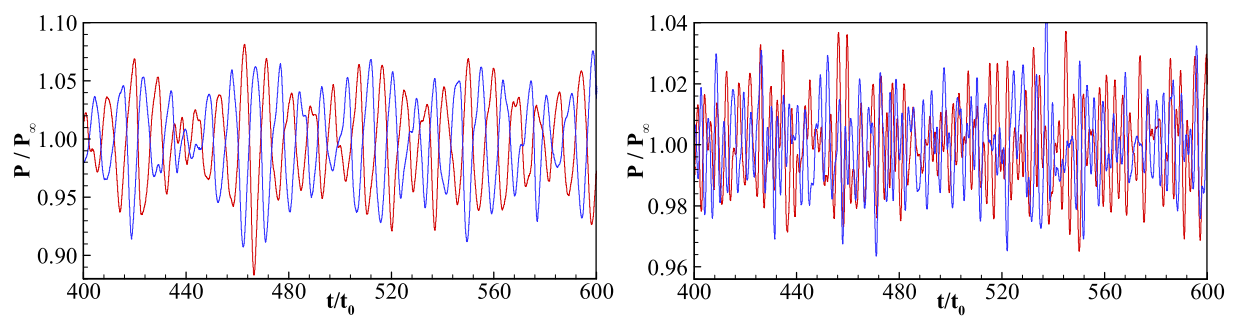

a)

b)

Fig. 20 Pressure history on either side of the jets at $(x / D=1, y / D=6, z / D=0)$ and $(x / D=-1, y / D=6$, and $z / D=0)$ : a) NPR $=5.60$, and b) $\mathrm{NPR}=9.34$. 


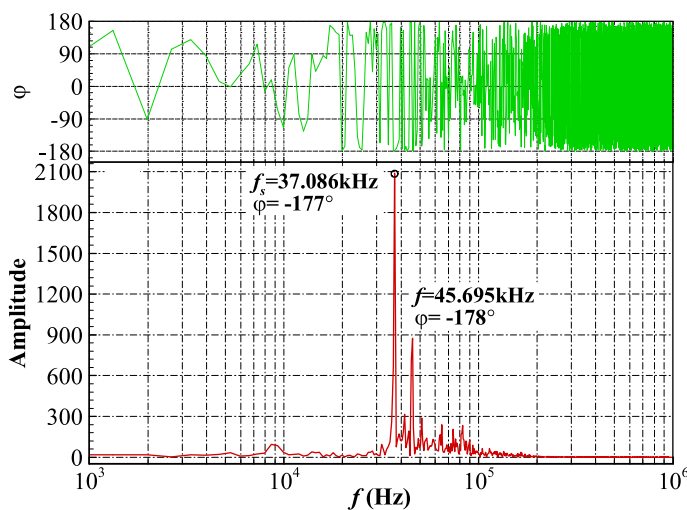

a)

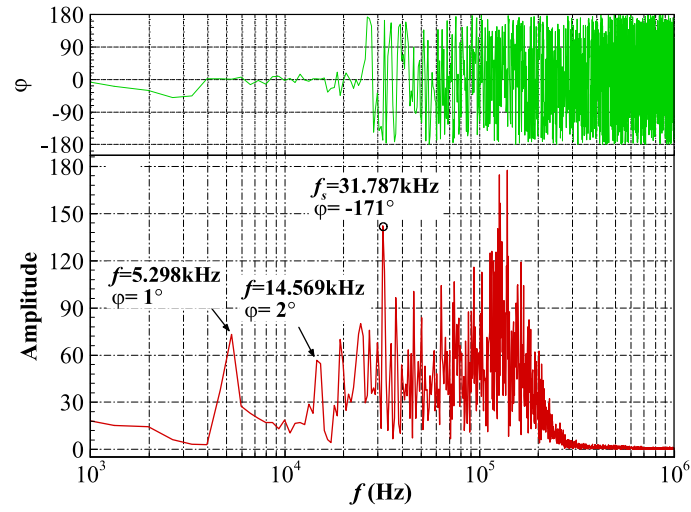

c)

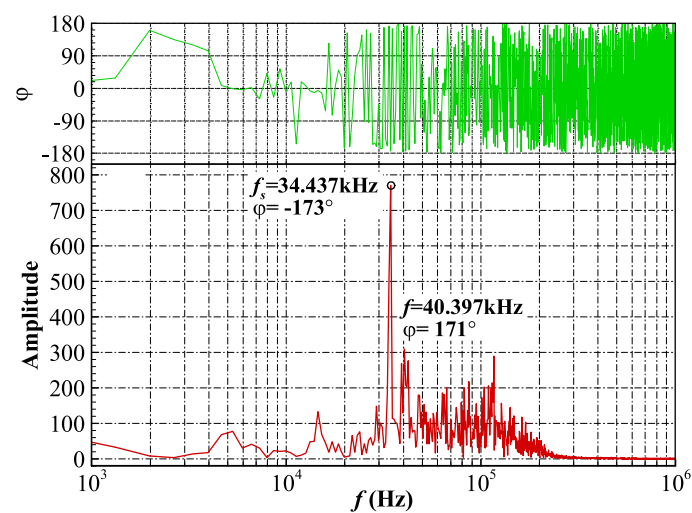

b)

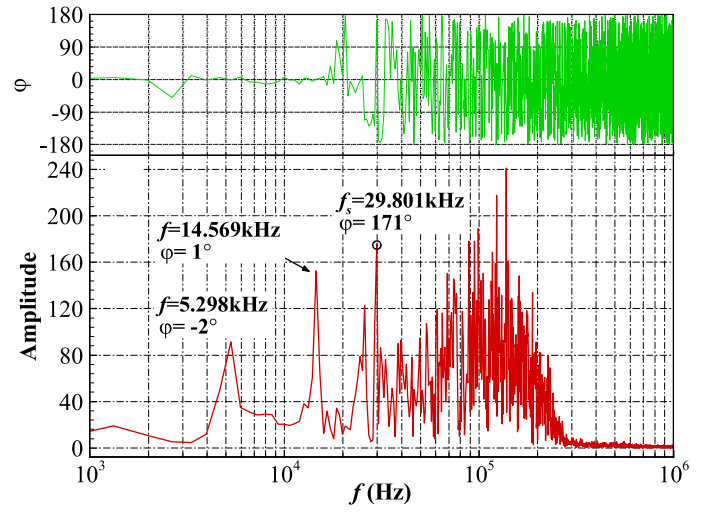

d)

Fig. 21 Cross spectrum and relative phase of pressure fluctuation on either side of the jets at $(x / D=1, y / D=6, z / D=0)$ and $(x / D=-1, y / D=6$, $z / D=0$ ): a) $\mathrm{NPR}=5.60$, b) $\mathrm{NPR}=7.47$, c) $\mathrm{NPR}=9.34$, and d) $\mathrm{NPR}=11.21$.

computed based on the fast Fourier transformation (FFT), as done by Gutmark et al. [23] and Panda and Seasholtz [25] based on measured pressure fluctuations as well as by Berland et al. [18] based on LES data. The corresponding amplitude and phase at $y / D=6$ for four different NPRs are plotted in Figs. 21a-21d as functions of the frequency.

The two signals of the pressure fluctuations (amplitude as functions of the frequency) at $x / D= \pm 1$ are perfectly matched; thus, only the spectrum at $x / D=1$ is presented in Fig. 21 . The dominant peak of $f_{s}=37.086 \mathrm{kHz}$ for NPR $=5.60$ has a phase angle of $-177 \mathrm{deg}$, corresponding to a single helical mode (azimuthal wave number $|m|=1$ ). Similar to NPR of 5.60, the dominant single helical mode for NPR $=7.47$ occurs at the screech frequency of $f_{s}=34.437 \mathrm{kHz}$ with $\varphi=-173 \mathrm{deg}$. Besides the screech tone, there is another peak in the spectrum, which is also helical with $f_{2 s}=45.695 \mathrm{kHz}, \varphi=-178 \mathrm{deg}$ for NPR $=5.60$ and $f_{2 s}=40.397 \mathrm{kHz}, \varphi=171 \mathrm{deg}$ for NPR $=7.47$. The amplitude of these frequency peaks is less than that of the corresponding shock screech tone and is generally referred to as the secondary shock screech tone [24]. Note that the multiple screech tones were also observed experimentally in underexpanded rectangular jets [므] and circular jets [71]. In particular, the fully expanded Mach number for the jets with multiple screech tones in [71] is around 1.8, which is close to the NPR $=5.60$ and 7.47 cases, as shown in Table 5 .

The shock screech tone can also be identified for two other higher NPRs, which is the single helical mode as well with $f_{s}=$ $31.787 \mathrm{kHz}, \varphi=-171 \mathrm{deg}$ for $\mathrm{NPR}=9.34$ and $f_{s}=$ $29.801 \mathrm{kHz}, \varphi=171 \mathrm{deg}$ for NPR $=11.21$. The secondary shock screech tone cannot be identified anymore. On the other hand, two peaks with lower frequencies of $f=5.298$ and $14.569 \mathrm{kHz}$ exist in the spectrum, which is the symmetric mode $(m=0)$ with a phase angle close to $0 \mathrm{deg}$. Note that these two lower frequencies also exist in the spectrum for NPR $=5.60$ and 7.47 at $y / D=2,4$, and 6 , but their amplitude is relatively small compared with that of the shock screech frequency. However, the amplitude of these two symmetric modes is larger than that of the shock screech tone at $y / D=2$ and 4 for NPR $=9.34$ and 11.21 and can be identified until at $y / D=8$. In addition, lots of discrete tones with amplitudes similar to that of the shock screech tone exist on the right side of the shock screech frequency. The higher frequency is generally consistent with the helical modes with larger azimuthal wave number, such as the double helical modes $(|m|=2)$. This means that the effects of helical modes

Table 5 Shock screech frequency and Strouhal number for different NPRs

\begin{tabular}{|c|c|c|c|c|c|c|c|c|c|c|}
\hline Author & Technique & NPR & $M_{j}$ & $f_{s}, \mathrm{kHz}$ & $f_{2 s}, \mathrm{kHz}$ & $S t_{s}^{\prime}$ & $S t_{2 s}^{\prime}$ & St & $S t_{s}^{\prime}, \%$ & $S t_{2 s}^{\prime}, \%$ \\
\hline Gutmark et al. [23] & Experiment & -- & 1.7 & -- & -- & 0.16 & -- & -- & -- & $-\ldots$ \\
\hline$[\overline{23}]$ & xperiment & -- & 1.9 & -- & -- & 0.12 & -- & -- & -- & \\
\hline tal. [32] & & 5.5 & -- & 217.80 & -- & 0.462 & -- & -- & -- & -- \\
\hline al. $[\overline{32}]$ & LES & 7.5 & -- & 179.61 & -- & 0.381 & -- & -- & -- & -- \\
\hline Presen & LES & 5.60 & 1.78 & 37.086 & 45.695 & 0.164 & 0.202 & 0.220 & -25.5 & -8.2 \\
\hline Prese & LE & 7.47 & 1.97 & 34.437 & 40.397 & 0.155 & 0.181 & 0.185 & -16.5 & -2.0 \\
\hline Present v & LES & 9.34 & 2.11 & 31.787 & -- & 0.146 & -- & 0.166 & -11.9 & -- \\
\hline Present work & LES & 11.21 & 2.23 & 29.801 & -- & 0.140 & -- & 0.152 & -7.9 & -- \\
\hline
\end{tabular}


of larger azimuthal wave number and higher frequency may increase greatly for NPR $=9.34$ and 11.21 .

Tam et al. $[15,16]$ derived the following screech tone frequency formula for a jet of Mach number $M_{j}$ using the multiple-scale shockcell mode and the adopted vortex sheet shock model:

$$
\begin{aligned}
S t & =\frac{f_{s} D_{j}}{U_{j}} \\
& =\frac{0.67}{\left(M_{j}^{2}-1\right)^{1 / 2}}\left[1+\frac{0.7 M_{j}}{\left[1+(\gamma-1) M_{j}^{2} / 2\right]^{1 / 2}}\left(\frac{T_{0}}{T_{\infty}}\right)^{1 / 2}\right]^{-1}
\end{aligned}
$$

where $S t$ is the Strouhal number; $f_{s}$ is the fundamental screech frequency; $M_{j}$ is the fully expanded jet Mach number; $U_{j}$ is the fully expanded jet velocity, both of which can be calculated according to the one-dimensional isentropic equations; and $D_{j}$ is the fully expanded jet diameter, which is related to the nozzle exit diameter $D$ by

$$
\frac{D_{j}}{D}=\left[\frac{1+(\gamma-1) M_{j}^{2} / 2}{1+(\gamma-1) M_{d}^{2} / 2}\right]^{(\gamma+1) / 4(\gamma-1)}\left(\frac{M_{d}}{M_{j}}\right)^{1 / 2}
$$

Panda [13] obtained a Strouhal number of 0.329 with $M_{j}=1.42$ based on measurements, which is $9 \%$ smaller than the prediction of 0.356 by Eq. (21). The Strouhal number obtained by Liu et al. [20] and Wang [72] using LES technique under the same flow condition are 0.415 and 0.385 , respectively, which are 15 and $8 \%$ larger than the prediction by Eq. (21).

The Strouhal numbers obtained by the present LES, with $S t_{s}^{\prime}$ and $S t_{2 s}^{\prime}$ being calculated based on the first and secondary shock screech frequency respectively, are compared with the empirical prediction by Eq. (21) in Table 5, which exhibits a similar level of error as Panda [13], Liu et al. [20], and Wang [72] (as for NPR $=5.60$ and 7.47, see $\overline{\Delta S} t_{2 s}^{\prime}$; the relations between the shock screech frequency $f_{s}$ and the secondary shock screech frequency $f_{2 s}$ will be discussed next). Note that the linearized small perturbation theory by Prandtl (as quoted by Panda [13] and Raman [17]) has been applied in the derivation of Eq. (21). Thus, Eq. (21) seems to work well only under relatively small jet Mach number $\left(1.0<M_{j}<1.8\right)$ [17], and most previous studies [16-22,24,25,70-72] on screech frequency deal with moderately underexpanded jets. For highly underexpanded jets, the shock screech frequency measurements with such high NPRs or $M_{j}$ are relatively rare. Therefore, the differences in flow structures of highly and moderately underexpanded jets are considered to be the main cause of deviation between the present LES and the prediction by Eq. (21). In addition, Gutmark et al. [23] provided a Stanton number of 0.16 for $M_{j}=1.7$ using the microphones for pressure fluctuations measurement, which is close to the value of 0.164 for $\mathrm{NPR}=5.60$ and $M_{j}=1.78$ obtained by the present LES. However, the Strouhal number for NPR $=5.5$ and 7.5 captured by La Vuorinen et al. [32] using LES approach are 0.462 and 0.381, which are two times larger than that obtained by the present LES as well as the prediction by Eq. (21) at similar NPR of 5.60 and 7.47. Note that the Strouhal number is defined as $S t=f D / 2 U_{1}$ in [32], and the corresponding screech frequencies are 217.8 and $179.614 \mathrm{kHz}$ with $D=0.0014 \mathrm{~m}$ and $U_{1}=330 \mathrm{~m} / \mathrm{s}$, which are one order larger than those obtained in the present study. This is probably caused by the differences in the simulation setup, and it is also implied that more effort needs to be devoted to screech frequency prediction of highly underexpanded jets.

From Fig. 21, the secondary shock screech frequency $f_{2 s}$ for NPR $=5.60$ and 7.47 is as helical as $f_{s}$. Table $\underline{5}$ indicates that the Strouhal number based on $f_{2 s}$ agrees better with the empirical prediction. This raises the question whether the higher peak frequencies of 45.695 and $40.397 \mathrm{kHz}$ for NPR $=5.60$ and 7.47 could be the shock screech frequencies. Hence, the numerical computations at these two NPRs are done a second time with nothing changed, and a comparison of the spectrum of pressure fluctuation between two independent runs is shown in Fig. 22. The signals of pressure fluctuation at two runs are perfectly matched, especially the value of the characteristic frequencies. More interestingly, the amplitude of frequency $45.695 \mathrm{kHz}$ is larger than that of $37.086 \mathrm{kHz}$ for NPR $=5.60$ in the second run, which indicates that the peak frequency of $45.695 \mathrm{kHz}$ turns into the shock screech frequency. Figure $22 \mathrm{~b}$ shows that this screech frequency switching phenomenon happens for NPR $=7.47$ as well. This is possibly due to the fact that the two modes are mutually exclusive, as observed by Raman [70] in a rectangular jet with a single-beveled nozzle exit with $M_{j}$ of 1.5 . And both the peak frequencies are valid shock screech frequencies for $\mathrm{NPR}=5.60$ and 7.47, and the jets switch the motion modes between the two helical modes of different dominant frequency randomly.

\section{E. Helicity Analysis}

The kinetic helicity is known as a global pseudoscalar, defined by the correlation between the vorticity and velocity as $H=\boldsymbol{\omega} \cdot \boldsymbol{u}$. Helicity can be either sign depending on the system of reference (leftor right-handed) and thus indicates the characteristics of local motions, i.e., helical $(H \gg 0$, clockwise; $H \ll 0$, counterclockwise) or nonhelical $(H \rightarrow 0)$ type. In addition, helicity is related with the formation of large-scale coherent structures in turbulent flows [73]. Thus, helicity of the jets is computed to reveal the helical characteristics of underexpanded jet shear layer to gain more intuitive insight into the instability.

Figure 23 shows the helicity on different cross-section planes of fully developed jets at different NPR. Numerous branches of smallscale unsteady helicity are generated after the nozzle exit; see, for example, $y / D=2$ for NPR $=7.47$. Those small leading branches usually turn up in pairs and form a well symmetric jet shear layer around the axis (i.e., the underexpanded jets behave with a dominant axisymmetric mode in the first shock cell). As the jet propagates downstream, the effect of helicity increases gradually, and helical modes become dominant in the structure of the jet shear layer; see, for example, $y / D=6$ for NPR $=5.60$. The dominant helical modes turn up in pairs, and the two branches rotate in the opposite direction.

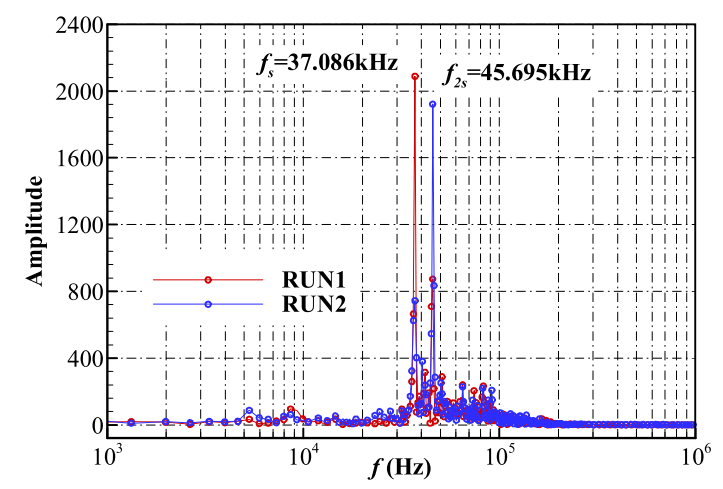

a)

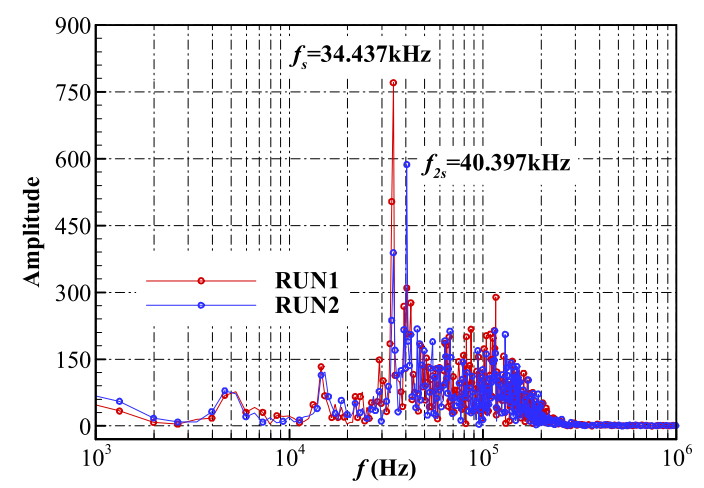

b)

Fig. 22 Spectrum of pressure fluctuation sampled near the jet shear layer at $x / D=1, y / D=6$, and $z / D=0$ during two independent computations at the same NPR: a) NPR $=5.60$, and b) NPR $=7.47$. 


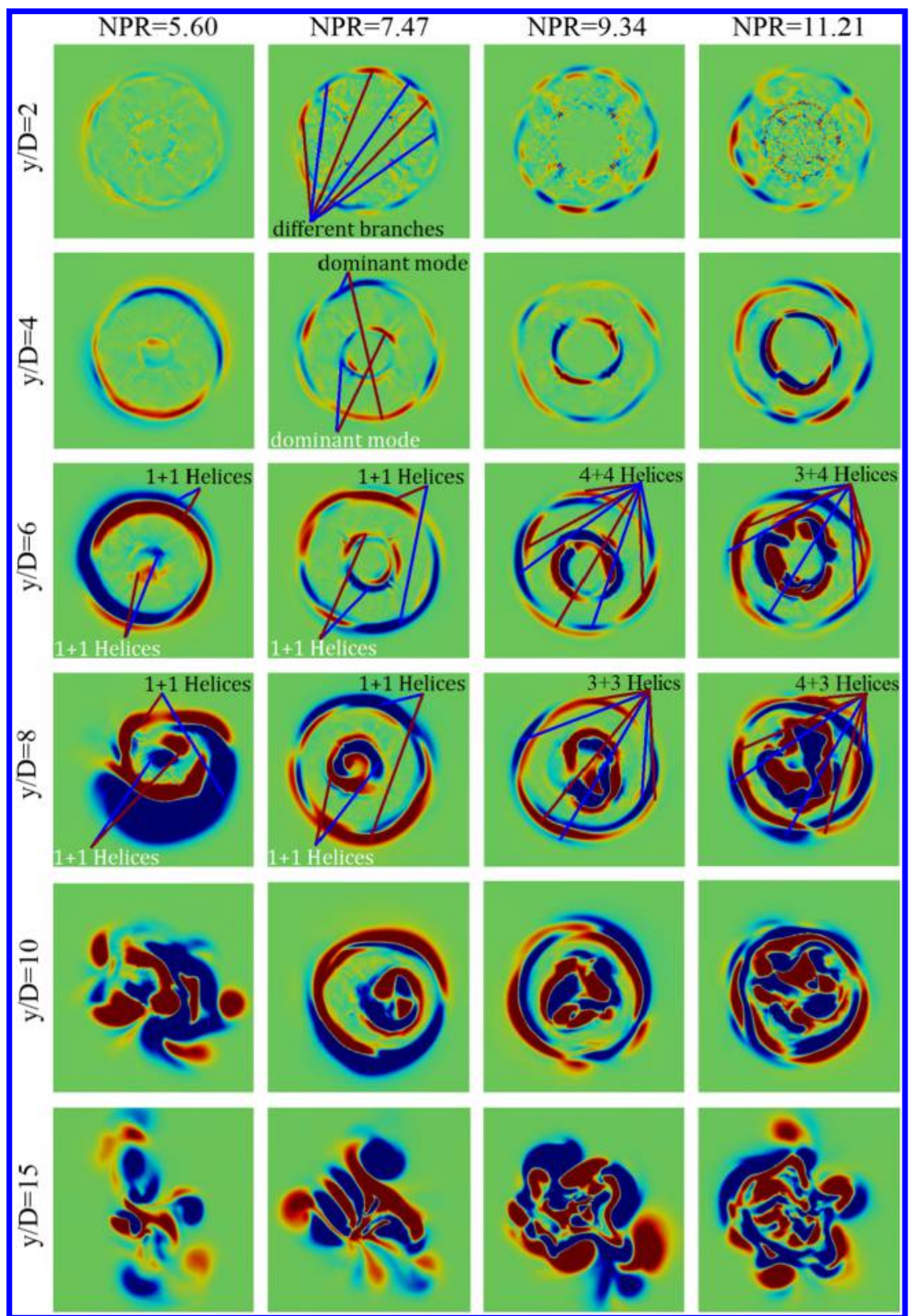

Fig. 23 Instantaneous snapshots of helicity on different cross-section planes of fully developed jets.

The three-dimensional motion in the near field of highly underexpanded sonic jets is the superposition of those different counter-rotating pairs of helical modes, rotating clockwise and counterclockwise alternatively. In the far downstream (e.g., $y / D>15)$, the dominant helical modes break up, and plenty of streamwise vortices are generated. These mean that the helicity plays a dominant role in the formation of near-field and downstream flow structures, which is consistent with the observations in previous numerical $[68,69]$ and experimental studies $[\underline{74}-76]$ on supersonic flows. It is also observed that the location from which the dominant helical modes originate increases as NPR increases, and the axial extent of the dominant helical mode is about $8 D$ for NPR $=5.60$ and slightly larger for higher NPRs.

One interesting finding is that the dominant helical modes in the outer shear layer exhibit the form of $1+1$ helices with opposite signs for NPR $=5.60$ and 7.47; see, for example, $y / D=4,6$, and 8 . Mattingly [77] found theoretically that the dominant disturbance in the very near field of the low-Reynolds-number axisymmetric jets is an axisymmetric one (azimuthal wave number $m=0$ ), whereas the single helical disturbance $(|m|=1)$ is dominant farther downstream. Danaila et al. [78] pointed out that the linear instability of round lowReynolds-number jets is characterized by two counter-rotating helical modes with azimuthal wave number of $|m|=1$. The single helical modes are also found to be dominant in low-Reynoldsnumber swirling jets by $\mathrm{Lu}$ et al. [79] and Oberleithner et al. [80] based on the proper orthogonal decomposition of LES data and particle image velocimetry measurements, respectively. Oberleithner et al. [80] indicated that two single helical modes (i.e., $m=1$ and $m=-1)$ have the same characteristic frequency and are depicted by one pair of counter-rotating helices. Besides the single helical modes, the double helical modes $(|m|=2)$ are also observed [80], which contain less energy and exhibit a quadrupole distribution. Note that the shock screech frequency is dominant in the spectrum and is consistent with the single helical modes. Therefore, the $1+1$ helices for NPR $=$ 5.60 and 7.47 resolved by helicity are mainly due to the superposition of two single helical modes $(m=1$ and $m=-1)$, and the corresponding frequency is the screech frequency, which switches randomly between the two peak frequencies of $f_{s}$ and $f_{2 s}$ because of the screech frequency switching phenomenon. However, the dominant helical modes for the other two higher NPRs look more complicated. For example, the dominant helical mode is $4+4$ at $y / D=6$ but switches to the form of $2+2$ helices at $y / D=10$ for $\mathrm{NPR}=9.34$. As stated previously, the effect of helical modes with larger azimuthal wave number on underexpanded jet shear layer increases greatly for 


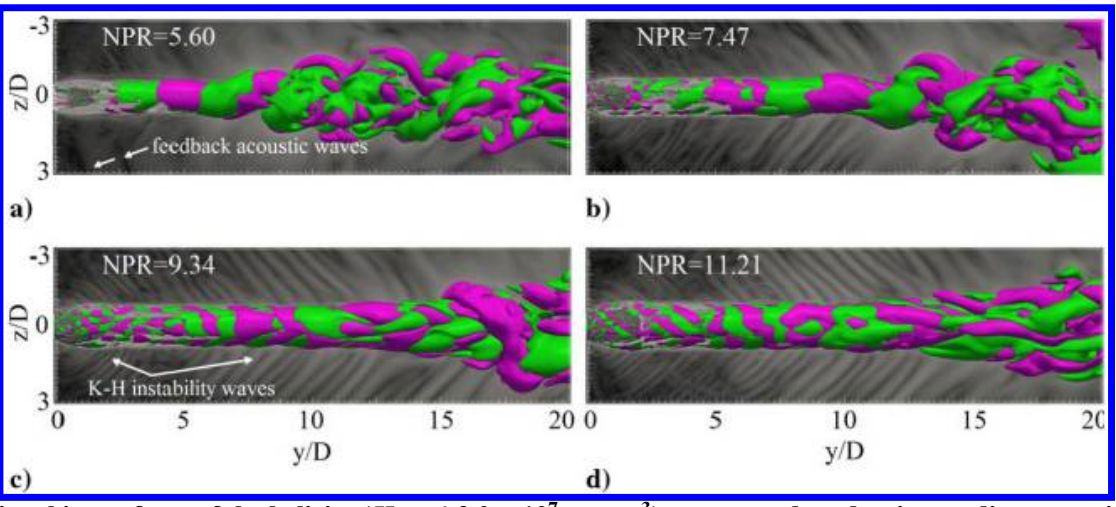

Fig. 24 Three-dimensional isosurfaces of the helicity $\left(H= \pm 2.0 \times 10^{7} \mathrm{~m} \cdot \mathrm{s}^{-3}\right)$ superposed on density gradient magnitude for different NPR.

$\mathrm{NPR}=9.34$ and 11.21, and it is their competition and superposition with the single helical mode $(|m|=1)$ that results in the multiple helices structure of jet shear layer at higher NPRs.

Figure 23 also indicates similar helical modes in the inner shear layer of the jets for all simulation cases. For example, the dominant helical modes in the inner shear layer take the same $1+1$ helices as that in the outer shear layer for NPR $=7.47$. Although the motion of the inner and outer shear layer are controlled by their own helicity independently, the instantaneous flowfield shows that both the inner and outer shear layer rotate in the same direction at the same time, clockwise or counterclockwise. At last, the isosurface of the helicity in Fig. 24 presents three-dimensional helical structures in the shear layer of underexpanded jets for different NPRs.

\section{F. Effect of Grid and Subgrid-Scale Model}

Figure 25 shows the helicity of the underexpanded jet at NPR of 5.60 on different cross-section planes obtained by the coarse mesh with the one-equation SGS model and the Smagorinsky model with the fine mesh respectively, whereas Fig. 26 presents the corresponding three-dimensional helical structures using the isosurfaces of helicity. The similar single helical mode is also captured by both the coarse mesh and the Smagorinsky model. It should be pointed out that the superposition of the two single helical modes (i.e., $m=1$ and $m=-1)$ may result in the $1+1$ helices as well the $2+2$ helices. Figure 23 indicates that the form of $1+1$ helices is captured by the fine mesh with the one-equation model. Figure 25a shows that the dominant helical mode captured by the coarse mesh with the oneequation model mainly exhibits the form of $2+2$ helices, such as at the locations of $y / D=4$ and 6 . On the other hand, the dominant helical mode obtained by the Smagorinsky model takes the $1+1$ helices, which is indicated by Fig. 25b at $y / D=4$ and 6 and is the same as that obtained by the one-equation model.

The dominant instability modes of the jet obtained by the coarse mesh and the Smagorinsky model are quantitatively identified using the cross spectrum and relative phase of pressure fluctuations in Fig. 27. As mentioned previously, the two peak frequencies obtained by the coarse mesh with the one-equation model are 35.761 and $44.370 \mathrm{kHz}$, respectively, which are close to that resolved by the fine mesh of 37.086 and $45.695 \mathrm{kHz}$. Both of the two peak frequencies are consistent with the single helical modes with a phase angle close to $180 \mathrm{deg}$. For the spectrum captured by the Smagorinsky model with the fine mesh, it is interesting to find that the two peak frequencies are the same as those obtained by the one-equation model (i.e., 37.086 and $45.695 \mathrm{kHz}$ ), which also indicates the single modes with a phase

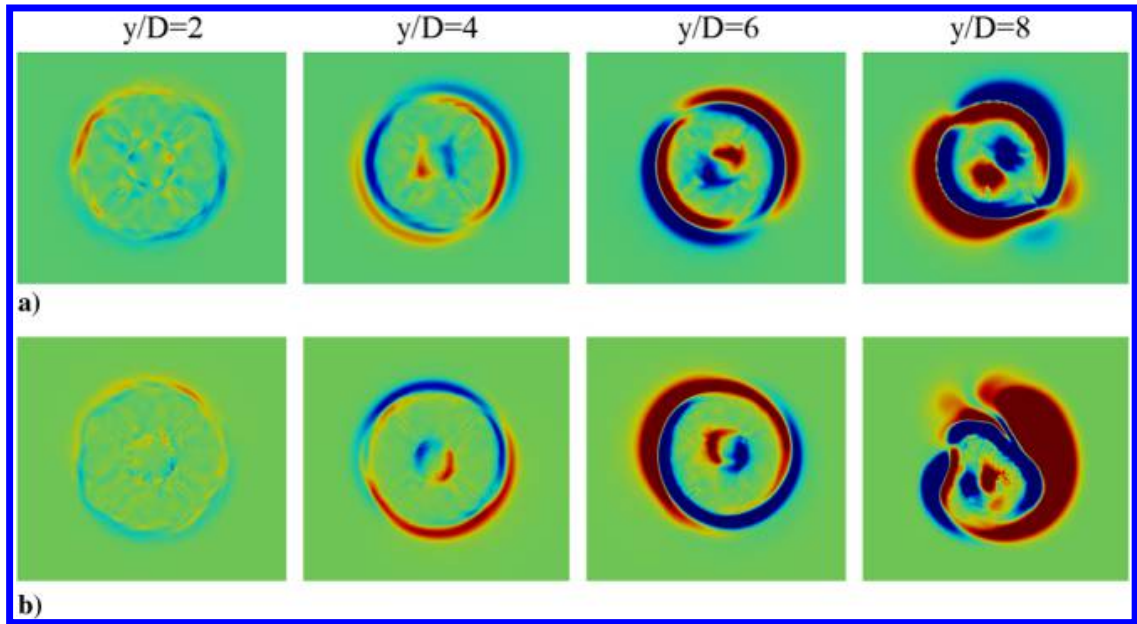

Fig. 25 Instantaneous snapshots of helicity on different cross-section planes for NPR = 5.60: a) coarse mesh with one-equation SGS model, and b) Smagorinsky model with fine mesh.

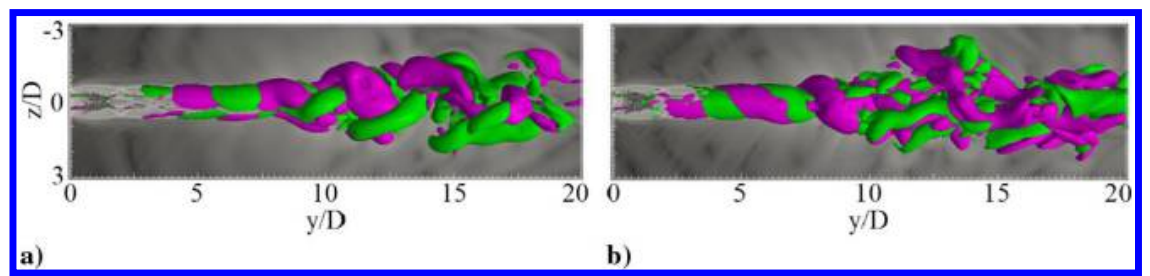

Fig. 26 Three-dimensional isosurfaces of the helicity $\left(H= \pm 2.0 \times 10^{7} \mathrm{~m} \cdot \mathrm{s}^{-3}\right)$ superposed on density gradient magnitude for NPR $=5.60$ : a) coarse mesh with one-equation SGS model, and b) Smagorinsky model with fine mesh. 


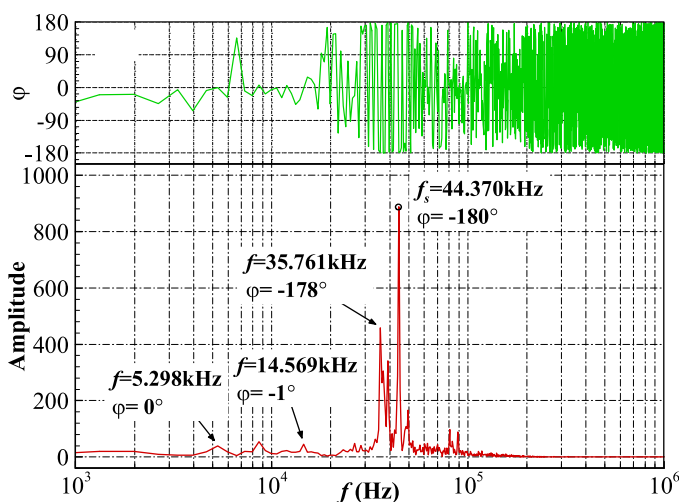

a)

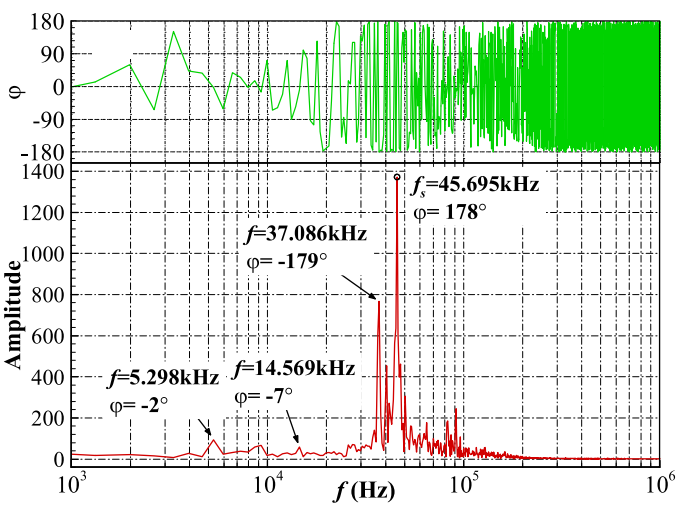

b)

Fig. 27 Cross spectrum and relative phase of pressure fluctuation on either side of the jets for NPR $=5.60:$ a) coarse mesh with one-equation SGS model, and b) Smagorinsky model with fine mesh.

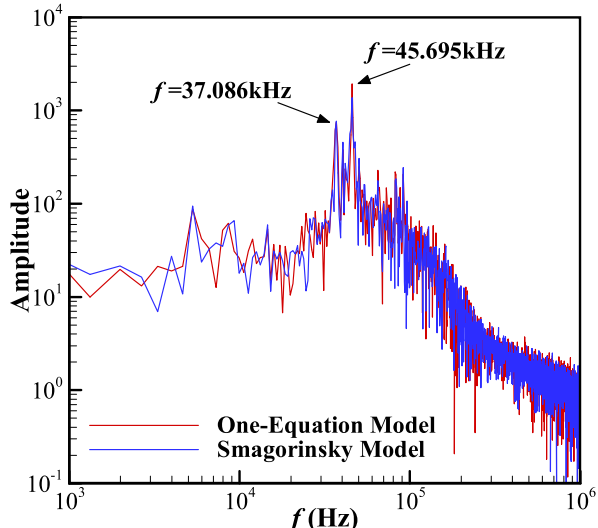

a)

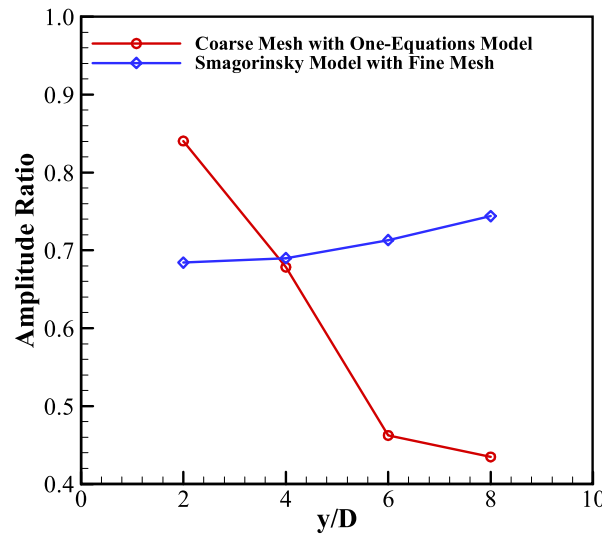

b)

Fig. 28 Representations of a) comparison between the spectrum of pressure fluctuation obtained by two different SGS models for NPR = 5.60, and b) nondimensional amplitude ratio of shock screech tone at different streamwise positions.

angle approximating $180 \mathrm{deg}$. More interestingly, the two symmetric modes of $f=5.298$ and $14.569 \mathrm{kHz}$, which are identified in the spectrum with different NPRs, also exist in the spectrums obtained by the coarse mesh and Smagorinsky model. The reason for the appearance of these two symmetric modes remains unresolved but is perhaps connected to the nozzle geometry design [17]

As mentioned previously, knowledge on the shock screech production of underexpanded jets has been gained owing to plenty of experimental and theoretical studies. Current understanding of the screech is primarily qualitative and quantitative predictions of the fundamental frequency at which the phenomenon occurs, whereas the information to determine the amplitude of the screech tone is limited [18]. Note that the higher peak frequencies of 44.370 and $45.695 \overline{\mathrm{kHz}}$ are screech frequencies for the coarse mesh and Smagorinsky model cases, respectively. Thus, the data based on the second run for NPR $=5.60$ with the fine mesh (i.e., RUN2, which is stated in Sec. IV.D) are employed here to maintain the consistency. Figure 28a compares the spectrum of pressure fluctuation for two different SGS models. It seems as if the effect of the Smagorinsky model is limited because the two signals are perfectly matched, except for the differences in amplitude of the peak frequencies. To examine quantitatively the effect of grid and SGS models, the shock frequency and the corresponding amplitude of pressure fluctuation sampled near the jet shear obtained by different simulation conditions are summarized in Table $\underline{6}$

From Table 6 , the amplitude of the screech tone obtained by the coarse mesh and the Smagorinsky model is smaller than that obtained by the fine mesh with the one-equation model. The amplitude of the screech tone obtained by the fine mesh with the one-equation model is chosen as the reference value, and the ratio between the amplitude obtained by the coarse mesh or the Smagorinsky model and the reference value is computed. The corresponding nondimensional results are plotted in Fig. $28 \mathrm{~b}$. The amplitude ratio for the coarse mesh at $y / D=2$ is around $\overline{0.84}$, which decreases greatly with the streamwise positions. This trend may be explained by the increase of dissipation due to the decay of the grid resolution along the streamwise direction. On the other hand, the amplitude ratio obtained using the Smagorinsky model is about 0.68 at $y / D=2$ and increases gradually to 0.74 at $y / D=8$. Figure 6 a indicates that the ratio between $k^{\text {sgs }}$ and TKE is relatively large in the near-field region and decreases gradually with increasing streamwise positions. The modeled SGS effects decrease along the streamwise direction accordingly. As mentioned previously, the dissipation of the Smagorinsky model is greater than that of the one-equation model. The more SGS effects it models, the greater deviation it makes. This

Table 6 Shock screech frequency and the corresponding amplitude of pressure fluctuations for $\mathrm{NPR}=\mathbf{5 . 6 0}$

\begin{tabular}{lccccc}
\hline \hline & & \multicolumn{4}{c}{ Amplitude } \\
\cline { 3 - 6 } Simulation conditions & $f_{s}, \mathrm{kHz}$ & $y / D=2$ & $y / D=4$ & $y / D=6$ & $y / D=8$ \\
\hline Fine mesh with & 45.695 & 219.26 & 435.07 & 1921.89 & 7523.53 \\
$\begin{array}{l}\text { one-equation model } \\
\begin{array}{l}\text { Coarse mesh with } \\
\text { one-equation model }\end{array}\end{array}$ & 44.370 & 184.25 & 295.16 & 888.49 & 3270.63 \\
$\begin{array}{l}\text { Smagorinsky model } \\
\text { with fine mesh }\end{array}$ & 45.695 & 150.01 & 300.18 & 1370.75 & 5596.64 \\
\hline \hline
\end{tabular}


could explain the trend for the Smagorinsky model in Fig. 28b. As a summary, it seems that the disadvantage of the Smagorinsky model on modeling the large-scale unsteadiness of underexpanded jets is not that remarkable as expected because of the excellent performances in capturing the peak frequencies. On the contrary, the Smagorinsky model has more advantages on computational efficiency because less equations are solved compared to the one-equation model. However, the one-equation model is recommended if the acoustic properties, such as the amplitude of the screech tone, are the issues to be focused on. It also appears that the grid resolution plays a more important role in revealing the dominant instability characteristics of underexpanded jets than the SGS models.

\section{Conclusions}

Large-eddy simulations of highly underexpanded jets with four different nozzle pressure ratios (NPR $=5.60,7.47,9.34$, and 11.21) are conducted to investigate the time evolution and instability characteristic of the jets. The performance of the equilibrium model, the Smagorinsky model, employed in LES to reveal the dominant instability characteristics is also investigated. A grid-convergence study is performed to validate the fidelity of the LES results. The time-averaged near-field properties of the jets are compared with the available literature data, and the reasonable agreement is observed. The main conclusions of this paper are summarized as follows.

The turbulent transition processes of highly underexpanded sonic jets with laminar inflow could be divided into four different flow stages (i.e., 1) the initial stage, 2) the laminar developing stage, 3) the turbulent transition stage, and 4) the fully turbulent stage). The turbulent transition processes are mainly characterized by the breaking down of the tip vortex ring, the loss of the flow symmetry, the distortion of the vortex rings, and the generation of streamwise vortices. Remarkable differences exist in the jet penetration in different flow stages. The jets propagate downstream slowly in the initial stage and speed up gradually once the turbulent transition takes place, accelerating more remarkably for higher NDR. The jet penetration coefficient $K 20$ exhibits larger values than 3.0 at higher NDR, which suggests that $K 20$ is a function of NDR rather than a constant. Short interim stages exist between two different flow stages, which is indicated by zigzag structures in the jet maximum width profiles. Analysis of the vorticity shows that both the dilatational and baroclinic terms, which are generally negligible in incompressible flows, become important in current supersonic flows. However, the effect of the baroclinic term on the distortion of the vortex rings in the near field is limited and not as large as expected, whereas it is still the vortex stretching term that plays the leading role.

The single helical mode corresponding to a phase angle close to \pm 180 deg is dominant for NPR $=5.60$ and 7.47 , whereas the effects of the double helical modes increase greatly for the higher NPR. There is some deviation in determining the shock screech frequencies between the current LES approach and previous empirical predictions, and this is possibly due to the great differences in flow structures. The shock screech frequency switching phenomenon, which indicates that both the discrete dominant peaks of $f_{s}$ and $f_{2 s}$ in the spectrum are valid shock screech frequencies, is observed for $\mathrm{NPR}=5.60$ and 7.47. The dominant instability characteristics of jets are directly visualized using helicity. Lots of unsteady helical branches of small scale are generated after the nozzle exit, and the dominant helical modes form gradually with the increasing streamwise positions. The dominant modes for NPR $=5.60$ and 7.47 exhibit the form of $1+1$ helices, whereas the complex and multiple helices for NPR $=9.34$ and 11.21 are due to an increase of the effect of double helical modes. The leading branches and the dominant helical modes usually turn up in pairs that rotate in the opposite direction. The three-dimensional motion of the underexpanded jets is the superposition of those different counter-rotating pairs, rotating clockwise and counterclockwise alternatively.

The dominant instability modes are also captured by both the coarse mesh and the Smagorinsky model, which exhibit the form of $2+2$ helices and $1+1$ helices, respectively. The peak frequencies obtained by the coarse mesh are slightly smaller than that resolved by the fine mesh, and the corresponding screech amplitude ratio decreases with the increasing streamwise positions because of a decay of grid resolution. The shock screech frequencies captured by the Smagorinsky model are the same as that obtained by the oneequation model, and the corresponding amplitude ratio of the shock screech increases with the streamwise positions. This behavior suggests that the disadvantage of the Smagorinsky model on modeling the instability characteristics of underexpanded jets is not as remarkable as expected, but the one-equation model is recommended if focusing on acoustic properties.

\section{Acknowledgments}

The project was supported by the Foundation for Innovative Research Groups of the National Natural Science Foundation of China (grant 10621202) and the National Natural Science Foundation of China (grant 11502270). The authors are also grateful to National Supercomputer Center in Tianjin for providing computational resources.

\section{References}

[1] Adamson, T. C., Jr., and Nicholls, J. A., "On the Structure of Jets from Highly Underexpanded Nozzles into Still Air,' Journal of the Aerospace Sciences, Vol. 26, No. 1, 1959, pp. 16-24.

[2] Crist, S., Sherman, P., and Glass, D., "Study of the Highly Underexpanded Sonic Jet," AIAA Journal, Vol. 4, No. 1, 1966, pp. 68-71.

[3] Donaldson, C. D., and Snedeker, R. S., "A Study of Free Jet Impingement. Part 1. Mean Properties of Free and Impinging Jets," Journal of Fluid Mechanics, Vol. 45, No. 2, Jan. 1971, pp. 281-319.

-[4] Chuech, S. G., Lai, M.-C., and Faeth, G. M., "Structures of Turbulent Sonic Underexpanded Free Jets," AIAA Journal, Vol. 27, No. 5, 1989 , pp. 549-559.

- [5] Wu, P.-K., Shahnam, M., Kirkendall, K. A., Carter, C. D., and Nejad, A. S., "Expansion and Mixing Processes of Underexpanded Supercritical Fuel Jets Injected into Superheated Conditions," Journal of Propulsion and Power, Vol. 15, No. 5, 1999, pp. 642-649.

[6] André, B., Castelain, T., and Bailly, C., "Experimental Exploration of Underexpanded Supersonic Jets," Shock Waves, Vol. 24, No. 1, 2014, pp. 21-32.

[7] Franquet, E., Perrier, V., Gibout, S., and Bruel, P., "Free Underexpanded Jets in a Quiescent Medium: A Review," Progress in Aerospace Sciences, Vol. 77, Aug. 2015, pp. 25-53.

- [8] Ouellette, P., and Hill, P. G., "Turbulent Transient Gas Injections," Journal of Fluids Engineering, Vol. 122, No. 4, 2000, pp. 743-753.

-[9] Otobe, Y., Kashimura, H., and Matsuo, S., "Influence of Nozzle Geometry on the Near-Field Structures of a Highly Underexpanded Sonic Jet," Journal of Fluids and Structures, Vol. 24, No. 2, 2008 , pp. 281-293.

[10] Menon, N., and Skews, B. W., "Shock Wave Configurations and Flow Structures in Non-Axisymmetric Underexpanded Sonic Jets," Shock Waves, Vol. 20, No. 3, 2010, pp. 175-190.

[11] Hatanaka, K., and Saito, T., "Influence of Nozzle Geometry on Underexpanded Axisymmetric Free Jet Characteristics," Shock Waves, Vol. 22, No. 5, 2012, pp. 427-434.

[12] Powell, A., "On the Mechanism of Choked Jet Noise," Proceedings of the Physical Society. Section B, Vol. 66, No. 12, 1953, pp. 1039-1056.

[13] Panda, J., "An Experimental Investigation of Screech Noise Generation," Journal of Fluid Mechanics, Vol. 378, Jan. 1999, pp. 71-96.

[14] Seiner, J. M., "Advances in High Speed Jet Aeroacoustics," 9th Aeroacoustics Conference, AIAA Paper 1984-2275, 1984.

[15] Tam, C. K. W., Seiner, J. M., and Yu, J. C., "Proposed Relationship Between Broadband Shock Associated Noise and Screech Tones," Journal of Sound and Vibration, Vol. 110, No. 2, 1986, pp. 309-321.

[16] Tam, C. K. W., "Supersonic Jet Noise," Annual Review of Fluid Mechanics, Vol. 27, 1995, pp. 17-43.

[17] Raman, G., "Advances in Understanding Supersonic Jet Screech: Review and Perspective," Progress in Aerospace Sciences, Vol. 34, No. 1, 1998, pp. 45-106.

[18] Berland, J., Bogey, C., and Bailly, C., "Numerical Study of Screech Generation in a Planar Supersonic Jet," Physics of Fluids, Vol. 19, No. 7, 2007, Paper 075105.

[19] Li, X. D., and Gao, J. H., "Numerical Simulation of the ThreeDimensional Screech Phenomenon from a Circular Jet," Physics of Fluids, Vol. 20, No. 3, 2008, Paper 035101. 
[20] Liu, J., Kailasanath, K., Ramamurti, R., Munday, D., Gutmark, E., and Lohner, R., "Large-Eddy Simulations of a Supersonic Jet and Its NearField Acoustic Properties," AIAA Journal, Vol. 47, No. 8, 2009, pp. 1849-1865.

-[21] Munday, D., Gutmark, E., Liu, J., and Kailasanath, K., "Flow Structure and Acoustics of Supersonic Jets from Conical Convergent-Divergent Nozzles," Physics of Fluids, Vol. 23, No. 11, 2011, Paper 116102.

[22] Tam, C. K. W., Parrish, S. A., and Viswanathan, K., "Harmonics of Jet Screech Tones," AIAA Journal, Vol. 52, No. 11, 2014, pp. 24712479.

[23] Gutmark, E., Schadow, K. C., and Bicker, C. J., "Mode Switching in Supersonic Circular Jets," Physics of Fluids, Vol. 1, No. 5, 1989, pp. 868-873.

[24] Powell, A., Umeda, Y., and Ishii, R., "Observations of the Oscillation Modes of Choked Circular Jets," Journal of the Acoustical Society of America, Vol. 92, No. 5, 1992, pp. 2823-2836.

- [25] Panda, J., and Seasholtz, R. G., "Measurement of Shock Structures and Shock-Vortex Interaction in Underexpanded Jets Using Rayleigh Scattering," Physics of Fluids, Vol. 11, No. 12, 1999, pp. 3761-3777.

-[26] Kawai, S., and Lele, K., "Large-Eddy Simulation of Jet Mixing in Supersonic Crossflows," AIAA Journal, Vol. 48, No. 9, 2010, pp. 2063-2083.

[27] Génin, F., and Menon, S., "Dynamics of Sonic Jet Injection into Supersonic Crossflow," Journal of Turbulence, Vol. 11, No. 4, 2010, pp. 1-30.

[28] Rana, Z. A., Thornber, B., and Drikakis, D., "Transverse Jet Injection into a Supersonic Turbulent Cross-Flow," Physics of Fluids, Vol. 23, No. 4, 2011, Paper 046103.

[29] Santiago, J. G., and Dutton, J. C., "Velocity Measurements of a Jet Injected into a Supersonic Crossflow," Journal of Propulsion and Power, Vol. 13, No. 2, 1997, pp. 264-273.

[30] Gorle, C., Gamba, M., and Ham, F., "Investigation of an Underexpanded Hydrogen Jet in Quiescent Air Using Numerical Simulations and Experiments," Center for Turbulence Research Annual Research Briefs, Stanford, CA, 2010

[31] Vreman, A. W., "An Eddy-Viscosity Subgrid-Scale Model for Turbulent Shear Flow: Algebraic Theory and Applications," Physics of Fluids, Vol. 16, No. 10, 2004, pp. 3670-3681.

[32] La Vuorinen, V., Yu, J., and Tirunagari, S., "Large-Eddy Simulation of Highly Underexpanded Transient Gas Jets," Physics of Fluids, Vol. 25, No. 1, 2013, Paper 016101.

[33] Greenshields, C. J., Weller, H. G., Gasparini, L., and Reese, J. M., "Implementation of Semi-Discrete, Non-Staggered Central Schemes in a Collocated, Polyhedral, Finite Volume Framework, for High-Speed Viscous Flows," International Journal of Numerical Methods in Fluids, Vol. 63, No. 1, 2010, pp. 1-21.

[34] Fureby, C., Chapuis, M., Fedina, E., and Karl, S., "CFD Analysis of the HyShot II Scramjet Combustor," Proceedings of the Combustion Institute, Vol. 33, No. 2, 2011, pp. 2399-2405.

[35] Chapuis, M., Fedina, E., and Fureby, C., "A Computational Study of the HyShot II Combustor Performance," Proceedings of the Combustion Institute, Vol. 34, No. 2, 2013, pp. 2101-2109.

[36] Arisman, C. J., Johansen, C. T., Galuppo, W. C., and McPhail, A., "Nitric Oxide Chemistry Effects in Hypersonic Boundary Layers," 43rd AIAA Fluid Dynamics Conference, AIAA Paper 2013-3104, 2013.

[37] Kurganov, A., and Tadmor, E., "New High-Resolution Central Schemes for Nonlinear Conservation Laws and Convection-Diffusion Equations," Journal of Computational Physics, Vol. 160, No. 1, 2000, pp. 241282.

[38] Jasak, H., Weller, H., and Gosman, A., "High Resolution NVD Differencing Scheme for Arbitrarily Unstructured Meshes," International Journal for Numerical Methods in Fluids, Vol. 31, No. 2, 1999, pp. 43149.

[39] Baba-Ahmadi, M. H., and Tabor, G., "Inlet Conditions for LES Using Mapping and Feedback Control," Computers and Fluids, Vol. 38, No. 6, 2009, pp. 1299-1311.

- [40] Chase, M. W., "JANAF Thermochemical Tables," Journal of Physical and Chemical Reference Data, Vol. 3, No. 2, 1974, pp. 311-480.

[41] Martin, M. P., Piomelli, U., and Candler, G. V., "Subgrid-Scale Models for Compressible Large-Eddy Simulations," Theoretical and Computational Fluid Dynamics, Vol. 13, No. 5, 2000, pp. 361-376.

[42] Fureby, C., and Möller, S. I., "Large Eddy Simulation of Reacting Flows Applied to Bluff Body Stabilized Flames," AIAA Journal, Vol. 33, No. 12, 1995, pp. 2339-2347.

[43] Tramecourt, N., Menon, S., and Amaya, J., "LES of Supercritical Combustion in a Gas Turbine Engine," 40th AIAA/ASME/SAE/ASEE Joint Propulsion Conference and Exhibit, AIAA Paper 2004-3381, July 2004.

[44] Smagorinsky, J., "General Circulation Experiments with the Primitive Equations: 1. The Basic Experiment," Monthly Weather Review, Vol.91, No. 3, 1963, pp. 99-164.
[45] Yoshizawa, A., "Statistical Theory for Compressible Turbulent Shear Flows, with the Applications to Subgrid Modeling," Physics of Fluids, Vol. 29, No. 7, 1986, pp. 2152-2164.

[46] Erlebacher, G., Hussaini, M. Y., Speziale, C. G., and Zang, T. A., "Toward the Large-Eddy Simulation of Compressible Turbulent Flows," Journal of Fluid Mechanics, Vol. 238, May 1992, pp. 155-185.

- [47] Yoshizawa, A., and Horiuti, K., "Statistically Derived Subgrid Scale Kinetic Energy Model for Large-Eddy Simulation of Turbulent Flows," Journal of the Physical Society of Japan, Vol. 54, No. 8, Aug. 1985, pp. 2834-2839.

[48] Menon, S., Yeung, P.-K., and Kim, W.-W., "Effect of Subgrid Models on the Computed Interscale Energy Transfer in Isotropic Turbulence," Computers and Fluids, Vol. 25, No. 2, 1996, pp. 165-180.

[49] Fureby, C., Tabor, G., Weller, H., and Gosman, A. D., "A Comparative Study of Sub Grid Scale Models in Homogeneous Isotropic Turbulence," Physics of Fluids, Vol. 9, No. 5, 1997, pp. 1416-1429.

[50] Fureby, C., Gosman, A. D., Tabor, G., Weller, H. G., Sandham, N., and Wolfshtein, M., "Large Eddy Simulation of Turbulent Channel Flows," Turbulent Shear Flows, Vol. 11, 1997, pp. 28-13.

[51] Chakravarthy, V., and Menon, S., "Large Eddy Simulations of Turbulent Premixed Flames in the Flamelet Regime," Combustion Science and Technology, Vol. 162, No. 1, 2001, pp. 175-222.

[52] Dauptain, A., Cuenot, B., and Gicquel, Y. M., "Large-Eddy Simulation of a Stable Supersonic Jet Impinging on Flat Plate," AIAA Journal, Vol. 48, No. 10, 2010, pp. 2325-2337.

[53] Pope, S. B., "Ten Questions Concerning the Large-Eddy Simulation of Turbulent Flows," New Journal of Physics, Vol. 6, No. 1, 2004, p. 35.

[54] Raman, G., and Rice, E. J., "Instability Modes Excited by Natural Screech Tones in a Supersonic Rectangular Jet," Physics of Fluids, Vol. 6, No. 12, 1994, pp. 3999-4008.

[55] Yang, M., "High Speed Pulsed Schlieren Technology and Its Application to Flow Visualization in Supersonic Combustion," M.S. Thesis, Inst. of Mechanics, Chinese Academy of Science, Beijing, 2012 (in Chinese).

[56] Ashkenas, H., and Sherman, F., "Structures and Utilization of Supersonic Free Jets in Low Density Wind Tunnels," NASA CR-60423, 1965.

[57] Ewan, B. C. R., and Moodie, K., "Structures and Velocity Measurements in Underexpanded Jets," Combustion Science and Technology, Vol. 45, Nos. 5-6, 1986, pp. 275-288.

[58] Dubief, Y., and Delcayre, F., "On Coherent-Vortex Identification in Turbulence," Journal of Turbulence, Vol. 1, No. 1, 2000, p. 11.

[59] Yuan, L. L., Street, R. L., and Ferziger, J. H., "Large-Eddy Simulations of a Round Jet in Crossflow," Journal of Fluid Mechanics, Vol. 379, Jan. 1999, pp. 71-104.

- [60] Drobniak, S., and Klajny, R., "Coherent Structures of Free Acoustically Stimulated Jet," Journal of Turbulence, Vol. 3, No. 3, 2002, p. N1.

[61] Poubeau, A., Paoli, R., Dauptain, A., Duchaine, F., and Wang, G., "Large-Eddy Simulations of a Single-Species Solid Rocket Booster Jet," AIAA Journal, Vol. 53, No. 6, 2015, pp. 1477-1491.

[62] Ouellette, P., "Direct Injection of Natural Gas for Diesel Engine Fueling," Ph.D. Thesis, Univ. of British Columbia, Vancouver, Canada, 1996.

[63] Hill, P. G., and Ouellette, P., "Transient Turbulent Gaseous Fuel Jets for Diesel Engines," Journal of Fluids Engineering, Vol. 121, No. 1, 1999, pp. 93-101.

[64] Rizk, W., "Experimental Studies of the Mixing Process and Flow Configurations in Two-Cycle Engine Scavenging," Proceedings of the Institution of Mechanical Engineers, Vol. 172, No. 1, 1958, pp. 417424.

[65] Diez, F. J., Sangras, R., Faeth, G. M., and Kwon, O. C., "Self-Preserving Properties of Unsteady Round Buoyant Turbulent Plumes and Thermals in Still Fluids," Journal of Heat Transfer, Vol. 125, No. 5, 2003, pp. 821-830.

[66] Grinstein, F. F., and DeVore, C. R., "Dynamics of Coherent Structures and Transition to Turbulence in Free Square Jets," Physics of Fluids, Vol. 8, No. 5, 1996, pp. 1237-1251.

[67] Husain, H. S., and Hussain, F., "Elliptic Jets Part 3. Dynamics of Preferred Mode Coherent Structure," Journal of Fluid Mechanics, Vol. 248, March 1993, pp. 315-361.

[68] Ingenito, A., and Bruno, C., "Physics and Regimes of Supersonic Combustion," AIAA Journal, Vol. 48, No. 3, 2010, pp. 515-525.

[69] Cecere, D., Ingenito, A., Giacomazzi, E., Romagnosi, L., and Bruno, C., "Hydrogen/Air Supersonic Combustion for Future Hypersonic Vehicles," International Journal of Hydrogen Energy, Vol. 38, No. 18, 2011, pp. 11969-11984.

- [70] Raman, G., "Screech Tones from Rectangular Jets with Spanwise Oblique Shock-Cell Structures," Journal of Fluid Mechanics, Vol. 330, Jan. 1997, pp. 141-168. 
71] Panda, J., Raman, G., and Zaman, K. B. M. Q., "Underexpanded Screeching Jets from Circular, Rectangular, and Elliptic Nozzles," $3 r d$ AIAA/CEAS Aeroacoustics Conference, AIAA Paper 1997-1623, 1997.

[72] Wang, G., "Large-Eddy Simulations of the Compressible Transverse Jet and Swirling Jet," Ph.D. Thesis, Univ. of Science and Technology of China, Hefei, PRC, 2012 (in Chinese).

[73] Yu, C., Xiao, Z., Shi, Y., and Chen, S., "Joint-Constraint Model for Large-Eddy Simulation of Helical Turbulence," Physical Review E, Vol. 89, No. 4, 2014, Paper 043021

[74] Hartfield, R. J., Hollo, S. D., and McDaniel, J. C., "Experimental Investigation of a Supersonic Swept Ramp Injector Using Laser Induced Iodine Fluorescence," Journal of Propulsion and Power, Vol. 10, No. 1, 1994, pp. 129-135.

[75] Riggins, D. W., and Vitt, P. H., "Vortex Generation and Mixing in ThreeDimensional Supersonic Combustors," Journal of Propulsion and Power, Vol. 11, No. 3, 1995, pp. 419-426.

[76] Schetz, J. A., Maddalena, L., and Throckmorton, R., "Complex Wall Injector Array for High-Speed Combustors," Journal of Propulsion and Power, Vol. 24, No. 4, 2008, pp. 673-680.
[77] Mattingly, G. E., "Unstable Waves on an Axisymmetric Jet Column," Journal of Fluid Mechanics, Vol. 65, No. 3, 1974, pp. 541-560.

[78] Danaila, I., Dušek, J., and Anselmet, F., "Direct Numerical Simulations of the Free, Unsteady, Round, Unforced Jet at Low Reynolds Numbers," Direct and Large-Eddy Simulation II, Vol. 5, ERCOFTAC Series, Springer, Dordrecht, The Netherlands, 1997, pp. 1-10.

[79] Lu, X., Wang, S., Sung, H.-G., Hsieh, S.-Y., and Yang, V., "Large-Eddy Simulations of Turbulent Swirling Flows Injected into a Dump Chamber," Journal of Fluid Mechanics, Vol. 527, March 2005, pp. 171195.

[80] Oberleithner, K., Sieber, M., Nayeri, C. N., Paschereit, C. O., Petz, C., Hege, H.-C., Noack, B. R., and Wygnanski, I., "Three-Dimensional Coherent Structures in a Swirling Jet Undergoing Vortex Breakdown: Stability Analysis and Empirical Mode Construction," Journal of Fluid Mechanics, Vol. 679, July 2011, pp. 383-414.

C. Bailly Associate Editor 
This article has been cited by:

1. Xiaopeng Li, Rui Zhou, Wei Yao, Xuejun Fan. 2017. Flow characteristic of highly underexpanded jets from various nozzle geometries. Applied Thermal Engineering 125, 240-253. [CrossRef]

2. Srisha M.V. Rao, Taiki Ikeda, Shingo Asano, Tsutomu Saito. 2017. Far-field hot-wire measurements on free jet from complex supersonic nozzles. Applied Thermal Engineering 118, 670-681. [CrossRef]

3. Xiaopeng Li, E. Fan, Wei Yao, Xuejun Fan. 2017. Numerical investigation of characteristic frequency excited highly underexpanded jets. Aerospace Science and Technology 63, 304-316. [CrossRef]

4. Wei Yao, Xuejun Fan. Development of zone flamelet model for scramjet combustor modeling . [Citation] [PDF] [PDF Plus]

5. Kun Wu, Peng Zhang, Wei Yao, Xuejun Fan. LES Study of Flame Stabilization in DLR Hydrogen Supersonic Combustor with Strut Injection . [Citation] [PDF] [PDF Plus]

6. Wei Yao, Yueming Yuan, Xiaopeng Li, Jing Wang, Xuejun Fan. A comparative study of elliptical and round scramjet combustors by Improved Delayed Detached Eddy Simulation . [Citation] [PDF] [PDF Plus] 